\title{
Controlling the SARS-CoV-2 spike glycoprotein conformation
}

\author{
Rory Henderson $\oplus^{1,2} \bowtie$, Robert J. Edwards ${ }^{1,2}$, Katayoun Mansouri', Katarzyna Janowska', \\ Victoria Stalls', Sophie M. C. Gobeil ${ }^{1}$ ', Megan Kopp', Dapeng Li', Rob Parks', Allen L. Hsu ${ }^{3}{ }^{3}$, \\ Mario J. Borgnia ${ }^{3}$, Barton F. Haynes ${ }^{1,2,4}$ and Priyamvada Acharya $\circledast^{1,5} \bowtie$
}

The coronavirus (CoV) spike (S) protein, involved in viral-host cell fusion, is the primary immunogenic target for virus neutralization and the current focus of many vaccine design efforts. The highly flexible S-protein, with its mobile domains, presents a moving target to the immune system. Here, to better understand S-protein mobility, we implemented a structure-based vector analysis of available $\beta$-CoV S-protein structures. Despite an overall similarity in domain organization, we found that S-proteins from different $\beta$-CoVs display distinct configurations. Based on this analysis, we developed two soluble ectodomain constructs for the SARS-CoV-2 S-protein, in which the highly immunogenic and mobile receptor binding domain (RBD) is either locked in the all-RBDs 'down' position or adopts 'up' state conformations more readily than the wild-type S-protein. These results demonstrate that the conformation of the S-protein can be controlled via rational design and can provide a framework for the development of engineered CoV S-proteins for vaccine applications.

$\mathrm{T}$ he ongoing global pandemic of the novel SARS-CoV-2 coronavirus presents an urgent need for the development of effective preventative and treatment therapies. The viral spike (S)-protein is a prime target for such therapies because of its critical role in the virus life cycle. The transmembrane CoV S-protein spike trimer is composed of interwoven protomers that include an N-terminal receptor-binding S1 subunit and a C-terminal S2 subunit that contains the fusion elements (Fig. 1a,b) ${ }^{1}$. The S1 subunit is subdivided into the N-terminal domain (NTD) followed by the receptor-binding domain (RBD) and two structurally conserved subdomains (SD1 and SD2). Together, these domains cap the S2 subunit, protecting the conserved fusion machinery. Binding to the host receptor via the RBD in $\mathrm{S} 1$ is followed by proteolytic cleavage of the spike by host proteases ${ }^{2}$. Large conformational changes in the S-protein result in S1 shedding and exposure of the fusion machinery in S2. Class I fusion proteins, such as the CoV-2 S-protein, undergo large conformational changes during the fusion process and must, by necessity, be highly flexible and dynamic. Indeed, cryo-EM structures of the SARS-CoV-2 spike reveal considerable flexibility and dynamics in the S1 subunit ${ }^{1,2}$, especially around the RBD, which exhibits two discrete conformational states-a 'down' state that is shielded from receptor binding and an 'up' state that is receptor-accessible.

The wealth of structural information for $\beta$-CoV S-proteins, including the recently determined cryo-EM structures of the SARS-CoV-2 spike ${ }^{1-11}$, has provided a rich source of detailed geometric information from which to begin precise examination of the macromolecular transitions underlying triggering of this fusion machine. Several structures of soluble ectodomain constructs that retain the complete S1 subunit and the surface-exposed S2 subunit have been determined. These include SARS-CoV-2 ${ }^{1,3}, \mathrm{SARS}^{4-8}$, MERS $^{4,9}$ and other human ${ }^{1,10}$ and murine ${ }^{11} \beta-\mathrm{CoV}$ S-proteins. These structures revealed remarkable conformational heterogeneity in the S-protein spikes, especially in the RBD region. Within a single protomer, the RBD could adopt a closed down state in which the RBD covers the apical region of the $S 2$ protein near the $C$ terminus of the first heptad repeat (HR1), or an open up state in which the RBD is dissociated from the apical central axis of S2 and the NTD (Fig. 1a). Furthermore, the cryo-EM structures strongly suggest a large degree of domain flexibility in both the down and up states in the NTD and RBD. Although these structures have provided essential information to identify the relative arrangement of these domains, the degree to which conformational heterogeneity may be altered via mutation during the natural evolution of the virus and in a vaccine immunogen design context remains to be determined.

In this study we quantify the variability in the S1 and S2 geometric arrangements to reveal important regions of flexibility to consider and to target for structure-based immunogen design. Based on these analyses, we design mutations that alter the conformational distribution of the domains in the S-protein. We visualize the effect of our designs using a structural determination pipeline relying first on single-particle analysis by negative-stain electron microscopy (NSEM) for rapid and low-cost assessment of the spike ectodomains at low resolution, followed by cryo-EM for high-resolution information on the changes introduced by these mutations. Our results reveal a heterogeneous conformational landscape of the SARS-CoV-2 spike that is highly susceptible to modification by the introduction of mutations at sites of contact between the S1 and S2 subunits. We also present data on modified SARS-CoV-2 ectodomain constructs stabilized in conformations that have not yet been seen in the current available structures, with great interest and direct application in vaccine design.

\section{Results}

Defining domain geometry and arrangements in SARS-CoV-2 spike. To characterize the unique arrangement of distinct domains

'Duke Human Vaccine Institute, Durham, NC, USA. ${ }^{2}$ Duke University, Department of Medicine, Durham, NC, USA. ${ }^{3}$ Genome Integrity and Structural Biology Laboratory, NIEHS, NIH, Department of Health and Human Services, Durham, NC, USA. ${ }^{4}$ Duke University, Department of Immunology, Durham, NC, USA. ${ }^{5}$ Duke University, Department of Surgery, Durham, NC, USA.凶e-mail: rory.henderson@duke.edu; priyamvada.acharya@duke.edu 
a
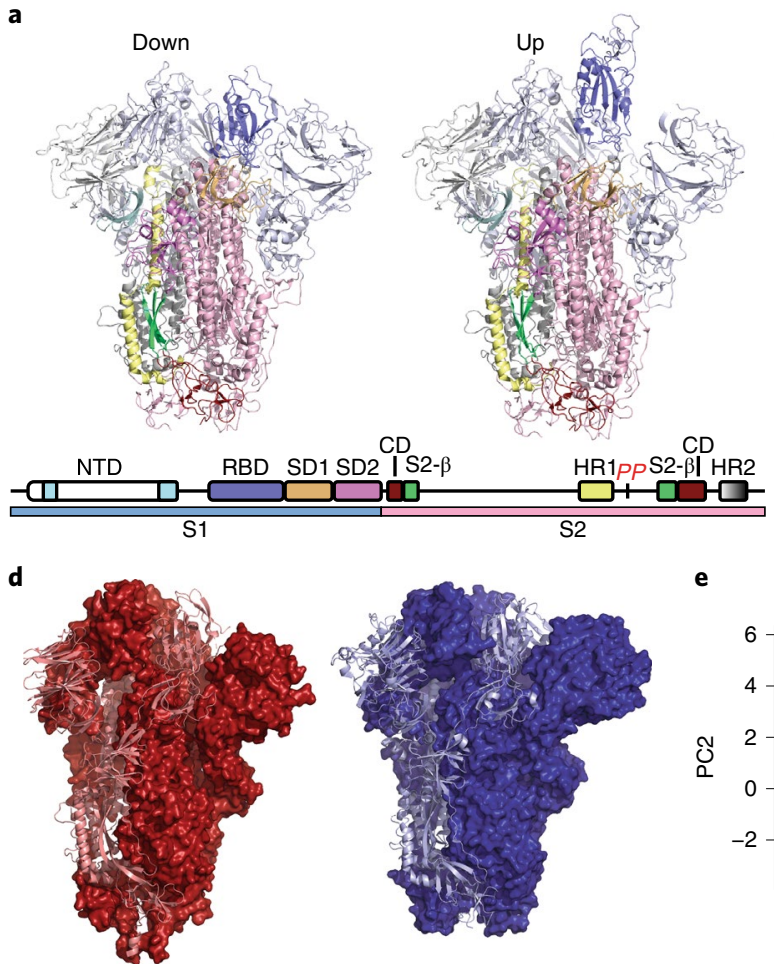

SARS-CoV-2

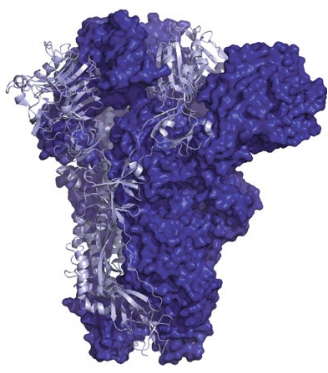

MERS b

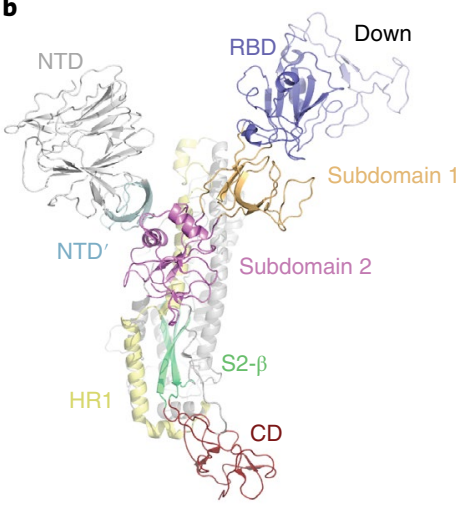

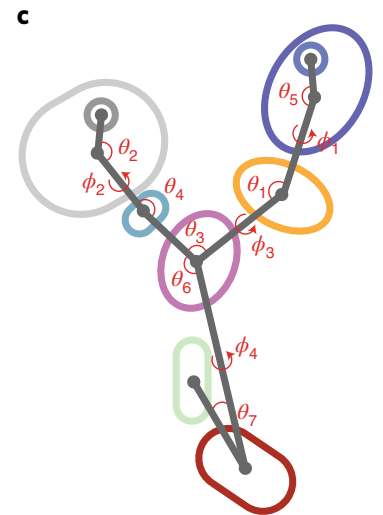

e

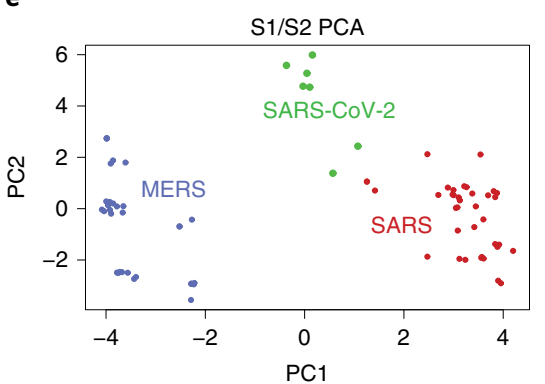

$\mathbf{f}$

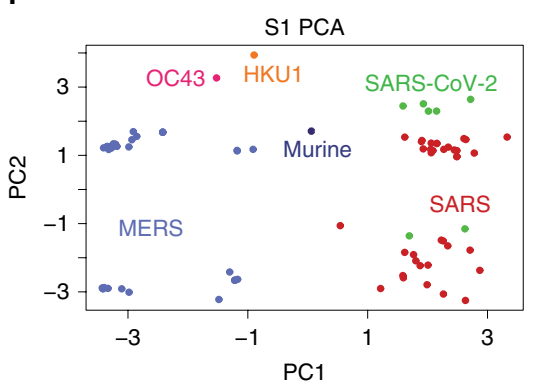

g
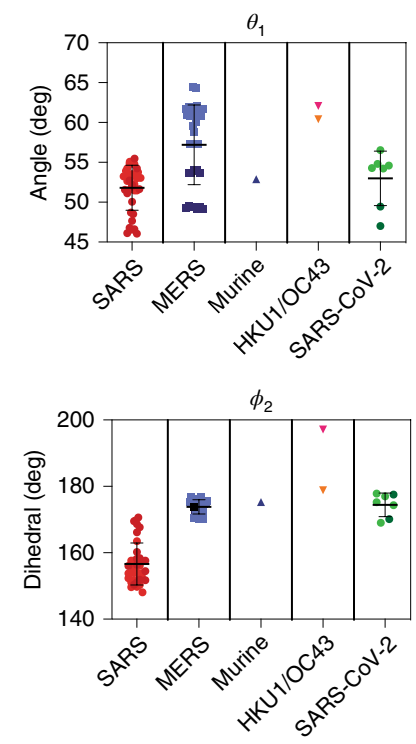

h

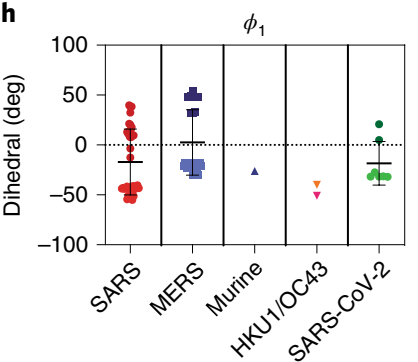

j

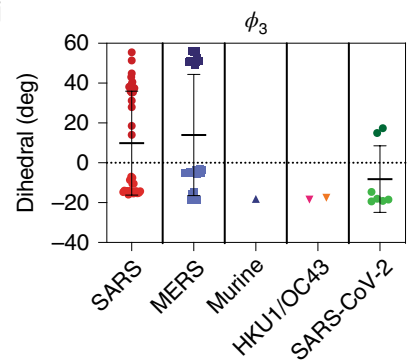

k

Fig. 1 | Vector-based analysis of the CoV S-protein demonstrates remarkable variability in the S-protein conformation within 'up' and 'down' states between CoV strains. a, Cartoon representations of the down (top left) and up (top right) state SARS-2 structures (PDB 6VXX and 6VYB, respectively), colored according to the specified domains (bottom). b, A single down-state protomer of the CoV S-protein with labeled domains (PDB 6VXX). The $\mathrm{RBD}$ is in its down conformation. $\mathbf{c}$, A simplified diagram of the CoV S-protein depicting the centroids and vectors connecting them, with the determined angles $(\theta)$ and dihedrals $(\phi)$ labeled. d, The SARS-2 (left, PDB 6VXX) and MERS (right, PDB 6Q04) structures, each with a single protomer depicted in cartoon representation and the remaining two in surface representation. The structures were aligned with the images captured from the same angle for visualization. e, Principal components analysis (PCA) of the SARS and MERS protomers showing measures between the S1 and S2 domains. f, PCA of the SARS, MERS, HKU1 and murine CoV protomers showing measures only between the S1 domains. $\mathbf{g}$, Angle between the SD2-to-SD1 vector and the SD1-to-RBD vector. h, Dihedral about the SD1-to-RBD vector. i, Dihedral about the NTD-to-NTD' vector. The OC 43 value of $-162^{\circ}$ has been adjusted to $197^{\circ}\left(+360^{\circ}\right)$ for visualization. $\mathbf{j}$, Dihedral about the SD2-to-SD1 vector. Data points for SARS, MERS and SARS-2 in g-j are colored according to the up (dark) and down (light) states and the color code in the PCA analysis in e and $\mathbf{f}$. Lines show mean and s.d. The PDB IDs for all structures represented in the PCA and angle/dihedral plots are listed in Supplementary Table 1. $\mathbf{k}$, Structural representation of the $\mathbf{g}-\mathbf{j}$ angles and dihedrals overlaid on an alignment between a SARS-2 down (cartoon structure with black centroids and lines; PDB 6VXX) and an up (ribbon structure with red centroids and lines; PDB 6VYB) state protomer. Adjacent protomers are depicted as a transparent surface with S1 (light blue) and S2 (light pink). Source data for graphs are provided in Supplementary Data 1. 
in the different $\beta$ - $\mathrm{CoV}$ spikes, we first developed a quantitative definition of their relative positions (Fig. 1). Examination of available SARS and MERS S-protein structures revealed the following: (1) domains in the S1 subunit (NTD, RBD and subdomains) and in the S2 subunit (connector domain (CD)) move as rigid bodies and (2) these domains display a pronounced array of relative shifts between those in the S1 subunit and the S2 subunit $\beta$-sheet motif and CD. To quantify these movements, we analyzed the relevant regions of motion and their structural disposition in available $\beta$-CoV ectodomain spike structures, including 15 for SARS ${ }^{4,5,7,8}, 16$ for MERS ${ }^{4,8,9,12}$, one each for $\mathrm{HKU}^{1,10}, \mathrm{OC} 43^{1,10}$ and a murine $\beta-\mathrm{CoV}$, and three SARS-CoV-2 ${ }^{3,13}$ structures (Fig. $1 \mathrm{e}-\mathrm{j}$ and Extended Data Fig. 1). Each protomer in these structures displaying asymmetric up/ down RBD states was examined independently, yielding a dataset of 83 structural states. Structures lacking the RBD were not analyzed. Only the S1 subunits were analyzed for the HKU1, OC43 and murine $\beta$-CoV S-protein structures. Information regarding whether the constructs are cleaved, mutated and/or liganded is provided in Supplementary Table 1.

The NTD was split into a primary N-terminal section and a secondary $\mathrm{C}$-terminal section based on visual inspection of this region in the various $\beta-\mathrm{CoV}$ structures. Vectors connecting each region's $\mathrm{C} \alpha$ centroids were generated and used to define the relative dispositions of the domains (Fig. 1b,c). The vector magnitudes and select angles and dihedrals were used to identify the breadth of differences in domain positioning and compare between $\beta-\mathrm{CoV}$ viruses (Fig. $1 \mathrm{e}-\mathrm{j}$ ). Projecting the S1 and S2 subunit vector data for SARS-CoV-1, MERS and SARS-CoV-2 using the principal components analysis method revealed that each virus's S-protein structures resided in distinct clusters (Fig. 1e). A similar analysis of the $S 1$ subunit vectors with the addition of data from the murine $\beta-\mathrm{CoV}$, HKU1 and OC43 generally retained these clusters, with the three additional structures lying in relatively unique positions (Fig. 1f). The SARS-CoV-1 structures split into two distinct clusters, separated largely by differences in the up/down states, with SARS-CoV-2 structures splitting with it. The distinct clustering of SARS-CoV-1 compared to SARS-CoV-2 in the PCA results, which include the S2 subunit data, suggests that there are key differences in the S1 disposition relative to S2 between SARS-CoV-1 and SARS-CoV-2.

Consistent with the PCA analysis, examination of specific angles and dihedrals indicated that $\beta$-CoV S-proteins in various viruses differ markedly from one another (Fig. $1 \mathrm{~g}-\mathrm{j}$ ). This is in addition to considerable variability in the domain arrangements within different virus S-proteins, demonstrating its wide structural lability (Fig. 1e-j). In particular, both $\phi_{1}$ and $\phi_{3}$ (Fig. 1h,j), describing the dihedrals about the vector connecting SD2 to SD1 and the vector connecting SD1 to the RBD (Fig. 1b,c), respectively, effectively reported on the up and down configurations and indicated substantial differences between SARS-CoV-1 and MERS in both the up and down states. Differences in the angular disposition of the NTD elements of SARS-CoV-1 and MERS as measured by $\phi_{1}, \theta_{2}$ and $\theta_{4}$ were also observed (Fig. $1 \mathrm{i}$ and Extended Data Fig. 1a,c). Additional S1 and S1/S2 subunit differences between viruses were observed for vectors involving SD2. This includes the SD2 to CD $\phi_{4}$ dihedral, which differs markedly between MERS/SARS-CoV-2 and SARS-CoV-1, and the angle between the vectors connecting the NTD' to SD2 and SD2 to the CD, demonstrating a shift in SARS-CoV-2 (Extended Data Fig. 1e,g). Finally, the disposition of the $\mathrm{CD}$ to the inner portion of $\mathrm{S} 2$ measured as an angle between a vector connected to an interior S2 $\beta$-sheet motif and the vector connecting the CD to SD2 indicates SARS-CoV-1 differs from both MERS and SARS-CoV-2 (Extended Data Fig. 1f). The MERS disposition appears to respond to RBD triggering in some structures, displaying a bimodal distribution (Extended Data Fig. 1f).

These results demonstrate that, although the individual domain architectures and overall arrangements are conserved, important a

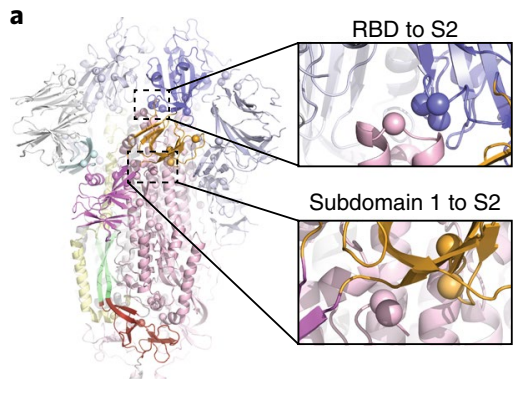

b SARS-COV-2 spike rS2d

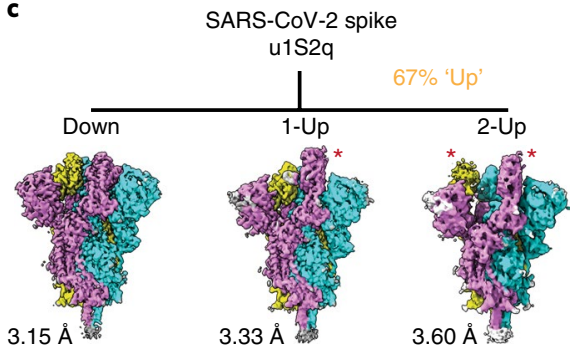

Fig. 2 | Cryo-EM structures reveal differential stabilization of the S-protein in the mutant ectodomain constructs. a, The S-protein spike, highlighting the two regions of interest for structure and computation-based design (PDB 6VXX). b, The rS2d RBD-to-S2 locked structure displaying only the all-RBD-down state map. c, The u1S2q SD1-to-S2 mutated structure displaying the all-RBD-down state, the 1-RBD up state and the 2-RBD up state. The percentage shown in orange is the sum of particles found in either the 1- or 2-RBD up state. Each protomer in each map is colored differently, with resolutions for these listed below. Red asterisks indicate up-state RBDs.

differences between these domains exist between viruses, suggesting that subtle differences in interdomain contacts could play a major role in determining these distributions and thereby alter surface antigenicity and the propensity of the domains to access up and down RBD states.

Differential stabilization of SARS-CoV-2 spike ectodomain RBD orientation. Based on the observed variability in the analysis of $\beta$-CoV spikes, we asked whether the propensity for the RBD to display the down and up states could be modified via mutations, without altering the exposed antigenic surfaces. We focused on domain pairs that moved relative to each other (Fig. 1) and identified interaction sites that could alter the S-protein conformational distribution. That is, modification of the up/down equilibrium could be achieved by modifying either the RBD or subdomain contact surfaces. To this end, we selected contact regions between the RBD and S2, the RBD and NTD, SD1 and S2, and SD2 and S2. Mutations at these sites were selected based on in silico mutagenesis using the Schrödinger Biologics suite $\mathrm{s}^{14-16}$. To eliminate exposure of the receptor binding site of the RBD, we examined the potential for disulfide linkages between the RBD and its contact with S2 near the C terminus of HR1 to prevent RBD exposure.

We identified a double cysteine mutant, S383C D985C (RBD to S2 double mutant (rS2d), Extended Data Fig. 2), as a candidate. The transition from the down state to the up state involves shifts in the RBD to NTD contacts. Therefore, to prevent these shifts, we identified a site in an RBD groove adjacent to the NTD, and prepared a triple mutant, D398L S514L E516L (RBD to NTD triple mutant (rNt), Extended Data Fig. 2). As SD1 acts as a hinge point for the RBD up/down transitions (Figs. $1 \mathrm{a}-\mathrm{c}$ and $2 \mathrm{i}, \mathrm{j}$ ), we hypothesized that enhanced hydrophobicity at the SD1 to S2 interface might shift the position of SD1, thus influencing the hinge and potentially the 

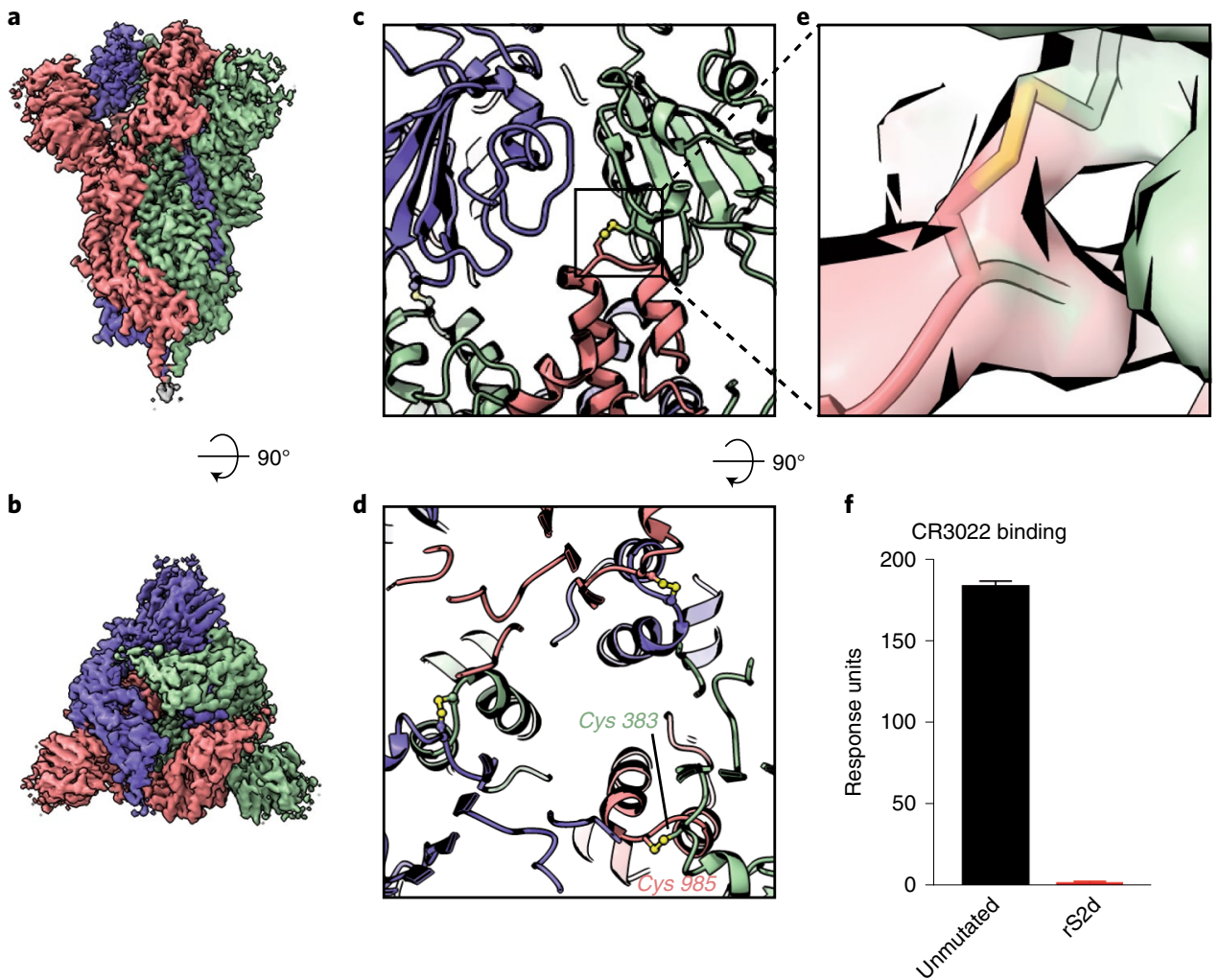

Fig. 3 | Cryo-EM structure of the rS2d construct locked in the down state. $\mathbf{a}$, Cryo-EM reconstruction of rS2d, colored by chain. $\mathbf{b}$, Rotated view (by $\sim 90^{\circ}$ ) of a. c. Magnified view showing the engineered disulfide linking the RBD of one protomer and the S2 domain of the adjacent protomer. The disulfide bridge is shown as spheres and colored by elements. $\mathbf{d}$, Rotated view (by $90^{\circ}$ ) of $\mathbf{c}$. e, Magnified view of the engineered interchain disulfide showing the cryo-EM density as a transparent surface and the underlying model in stick representation. f, Binding of antibody CR3022 to the unmutated structure (black bar) and rS2d (red bar). The spike was captured on a streptavidin chip and binding to CR3022 immunoglobulin-G (IgG) was measured by surface plasmon resonance (SPR). Data shown are means and s.d. of three technical repeats and are representative of five independent experiments.

propensity for RBD triggering. A double mutant, N866I A570L (subdomain 1 to S2 double mutant (u1S2d)), and a quadruple mutant, A570L T572I F855Y N856I (subdomain 1 to S2 quadruple mutant (u1S2q)), were identified for this purpose (Extended Data Fig. 2). Finally, we identified a double cysteine mutant, G669C and T866C, to link SD2 to S2 (subdomain 2 to S2 double mutant (u2S2d), Extended Data Fig. 2).

NSEM analysis of SARS-CoV-2 spike ectodomain proteins. The mutants were prepared in the context of a previously published SARS-CoV-2 ectodomain construct ${ }^{3}$ (Extended Data Fig. 3). To assess the quality of the purified proteins, we performed NSEM analysis (Extended Data Fig. 4). The micrographs show a reasonably uniform distribution of particles consistent with the size and shape of the SARS-CoV-2 spike ectodomain. Two-dimensional (2D) class averages showed spike populations with well-resolved domain features.

Following 3D classification then homogeneous refinement, the unmutated spike resolved into two classes of roughly equal proportions that differed in the position of their RBD domains. One class displayed all three RBDs in their down positions, whereas the other class displayed one RBD in the up position. This was consistent with published cryo-EM results ${ }^{13}$ that describe a 1:1 ratio between the down and 1-up states of the SARS-CoV-2 spike ectodomain.

The mutant spikes, analyzed using a similar workflow, showed different conformation distributions compared to the unmutated spike. The most pronounced changes were observed in the rS2d and u1S2q constructs (Extended Data Fig. 4c,e). For the rS2d construct, we observed only the down conformation (Extended Data Fig. 4c); the 1-RBD up state was not found in this dataset. In the u1S2q data, we observed an S-protein population with 2 RBDs in the up position, which has been reported before for the SARS-CoV-1 and MERS $\mathrm{CoV}$ spike ectodomains ${ }^{7,9}$. These two constructs were selected for downstream, high-resolution characterization by cryo-EM.

Cryo-EM and properties of the SARS-CoV-2 spike constructs. We collected cryo-EM datasets for the rS2d and u1S2q constructs (Figs. 2-6, Table 1 and Extended Data Figs. 5-9). Consistent with the NSEM analysis, following multiple rounds of $2 \mathrm{D}$ and $3 \mathrm{D}$ classification, we found a population of down state spike in the rS2d dataset (Fig. 3 and Extended Data Fig. 6). We then implemented additional exhaustive ab initio classifications, as well as heterogeneous classifications using low-pass filtered maps of known open conformations of $\mathrm{CoV}$ spikes to search for open state spikes in the dataset. We were unable to find any such states, suggesting that the disulfide bond between the RBD and the S2 subunit effectively locked the SARS-CoV-2 spike in its down conformation. The rS2d cryo-EM reconstruction had an overall resolution of $2.7 \AA$, but the local resolution varied widely (Extended Data Fig. 6g), with the highest resolutions in the S2 subunit (Extended Data Fig. 6h) and higher disorder and lower local resolutions in the S1 subunit; this is consistent with previous structures of the SARS-CoV-2 spike s.13 $^{3,}$ We observed connecting density at the site of the engineered disulfide link in rS2d, thus confirming disulfide formation (Fig. 3c-e). Alignment of this structure with that of the unmutated down closed state structure $(\mathrm{PDB} 6 \mathrm{VXX})^{13}$ indicated that the overall protein structure was otherwise unperturbed (root-mean-square deviation (r.m.s.d.) of $0.5 \AA$ ). 
a

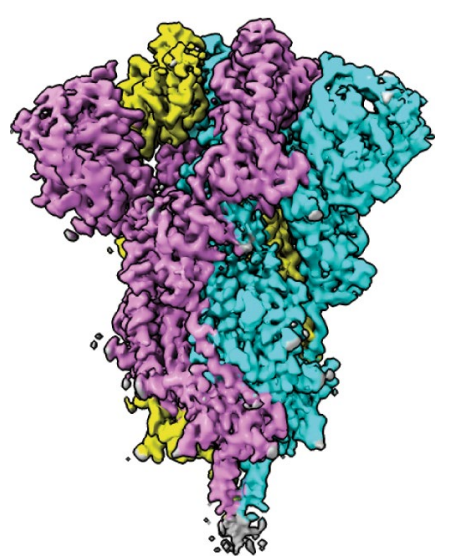

c

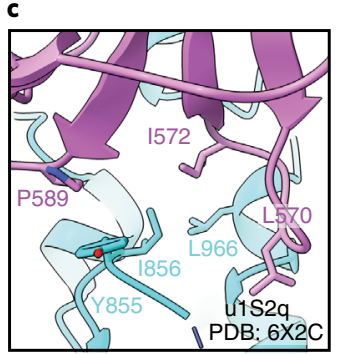

d

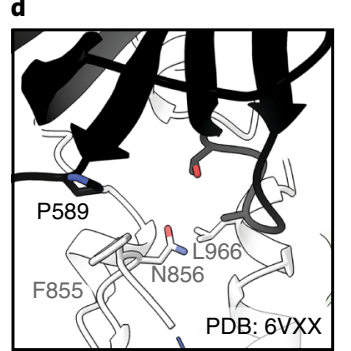

e

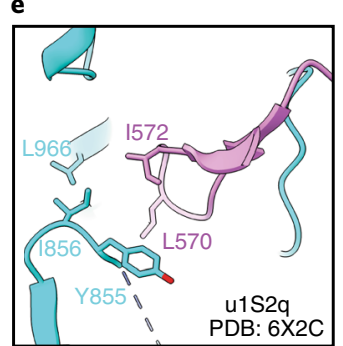

f

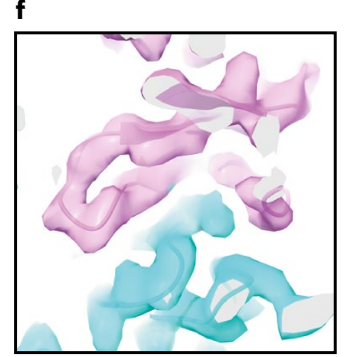

b

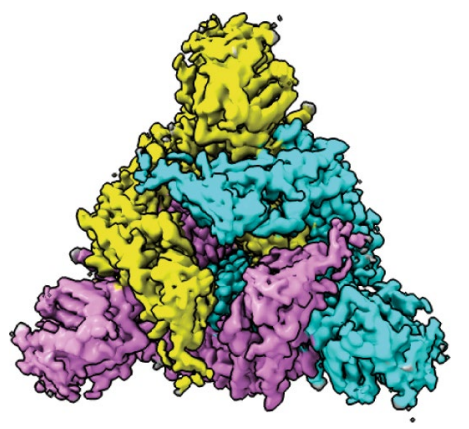

g

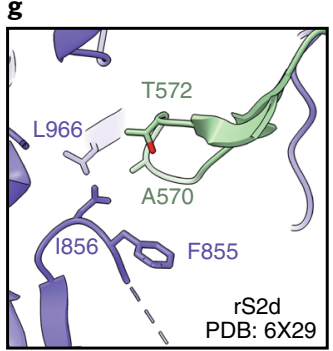

h

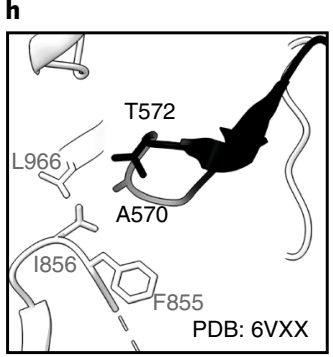

i
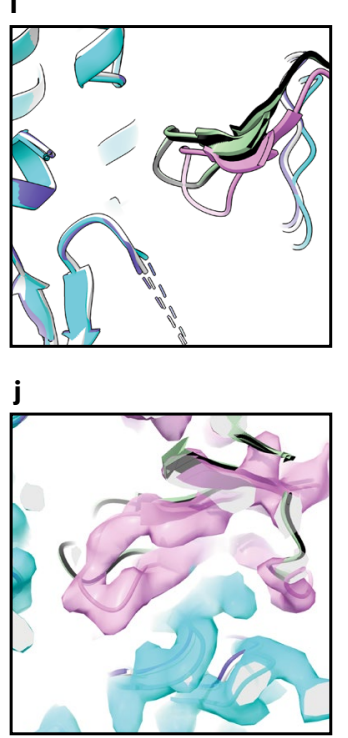

Fig. 4 | Cryo-EM structures of RBD-down S-proteins reveal differential stabilization of domain positions. a, Cryo-EM reconstruction of u1S2q colored by chain. $\mathbf{b}$, Rotated view (by $\sim 0^{\circ}$ ) of a. c. Magnified view of the region containing the mutations, showing the proximity of the F855Y and N856I residue loop to the S2 residue L966 and S1 residue P589. d, Similar region to that shown in c but for the unmutated structure (PDB 6VXX). e, Magnified view of the region containing the mutations at a different angle than the one shown in c. f, The loop containing the A570L and T572I mutations, with the cryo-EM map shown as a transparent surface and fitted model shown in cartoon representation. $\mathbf{g}, \mathbf{h}$, Similar region to that shown in $\mathbf{e}$ but for the rS2d structure (PDB 6X29) (g) and the unmutated structure (PDB 6VXX) (h). i,j, Overlay of the three down state structures (i) and with the cryo-EM reconstruction for the down state for the u1S2q construct $(\mathbf{j})$.

We next tested the binding of rS2d to antibody CR3022 (Fig. 3f and Extended Data Fig. 7a) ${ }^{17,18}$. CR3022 binds an epitope on the RBD of the SARS-CoV- 1 and SARS-CoV-2 spikes that is occluded in the down RBD conformation. Although we observed robust binding for the unmutated spike to CR3022, no measurable binding was observed for rS2d, confirming that the RBD in the rS2d construct is locked in a down conformation. Also, as expected, the rS2d mutant showed reduced binding to angiotensin-converting enzyme 2 (ACE-2) compared to the unmutated spike (Extended Data Fig. 7 b,c), and such activity could be abrogated using an ACE-2 or a CR3022 IgG-immobilized column for purification (Extended Data Fig. 7c-h).

In contrast to $\mathrm{rS} 2 \mathrm{~d}$, the $\mathrm{u} 1 \mathrm{~S} 2 \mathrm{q}$ spike displayed widespread rearrangement of the S1 subunits (Extended Data Figs. 8 and 9). In the down state structure, the mutated S2 position remained in the configuration observed in the unmutated construct, with the F855Y and N856I residue loop in close proximity to S2 residue L966 and S1 residue P589 (Fig. 4c,d), suggesting that these mutations had little impact on the observed shifts. However, the S2-interactive SD1 displayed a rigid body movement relative to both the $\mathrm{rS} 2 \mathrm{~d}$ and unmutated constructs, with $\theta_{1}$ and $\phi_{3}$ displacements of $3.7^{\circ}$ and $2.4^{\circ}$, respectively (Fig. $4 \mathrm{e}-\mathrm{j}$ and Extended Data Fig. 10). This resulted in displacement of the A570L + T572I-containing loop from the unmutated position near the S2 L966 residue (Fig. 4i,j). The S2 contact disruption is accompanied by an angular shift of the NTD away from the primary trimer axis due to SD1 to NTD' contacts, yielding $\theta_{3}$ and $\phi_{2}$ shifts of $6.0^{\circ}$ each, respectively (Extended Data Fig. 10). The subdomain rearrangement impacts the positioning of the RBD with only a minor shift in the $\phi_{1}$ dihedral of $0.1^{\circ}$ indicating that the RBD moved with SD1, indicated in the $\theta_{1} / \phi_{3}$ shifts. The newly acquired arrangement in both the RBD and NTD was further accompanied by an apparent increase in their flexibility, suggesting conformational heterogeneity. These down state shifts were observed in both the single RBD up structure and the two RBD up structures (Figs. 5 and 6 and Extended Data Fig. 10). The extent to which the SD1 shift differed from that observed in the unmutated construct was context-dependent in the 1-RBD up state (Fig. 5c-h). Although the down state RBD in contact with the up state RBD displayed the large shift in position observed in the all down state (Fig. $5 \mathrm{~d}, \mathrm{~g}$ ), the down state RBD with its terminal position free displayed an 


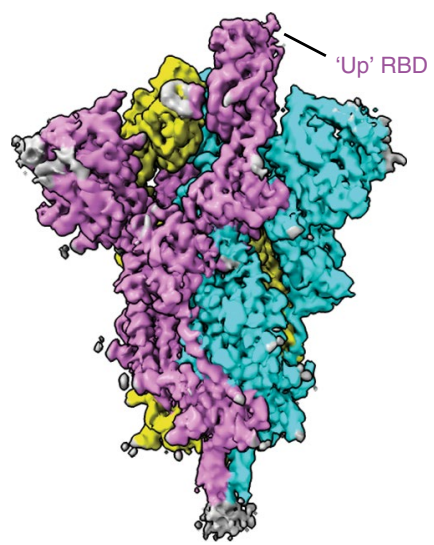

d

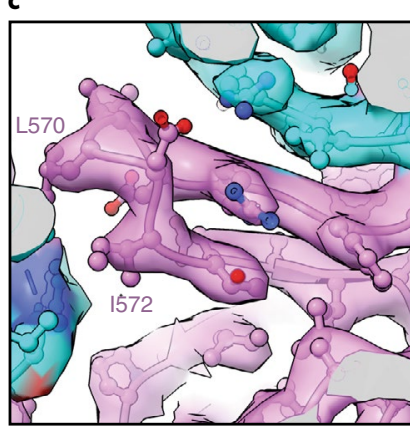

f

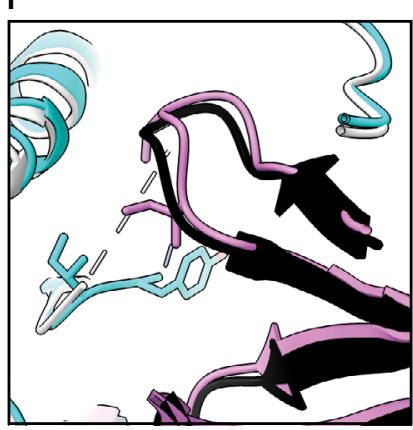

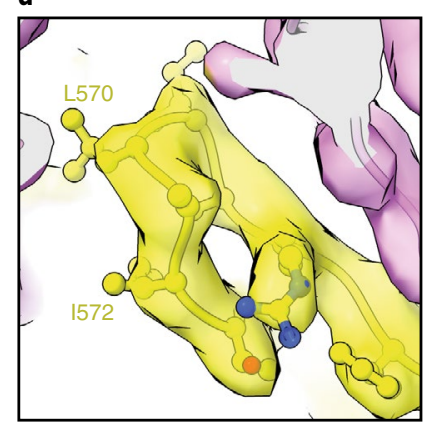

g

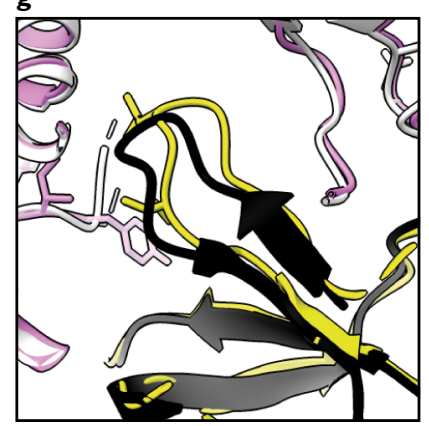

b

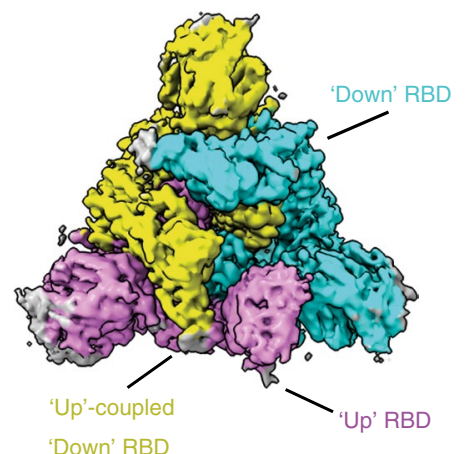

e

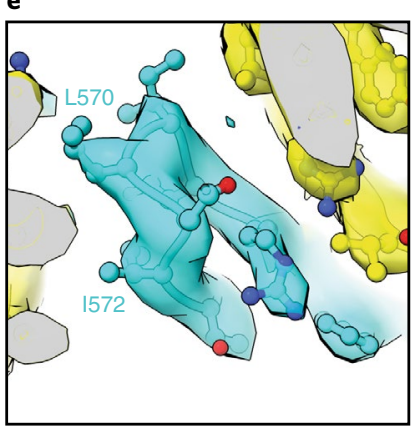

$\mathbf{h}$

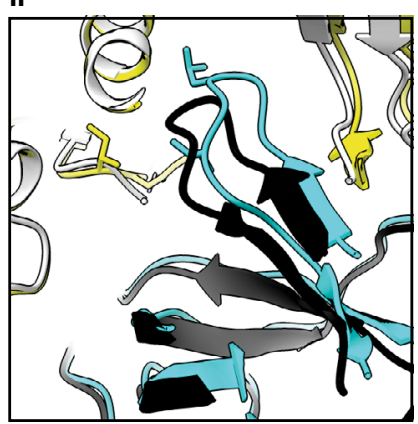

Fig. 5 | Cryo-EM structure of the u1S2q 1-RBD up state reveals increasing relaxation of the triggered RBD toward the unmutated structure. a, Cryo-EM reconstruction of the u1S2q 1-RBD up state colored by chain. b, Rotated view (by $\sim 90^{\circ}$ ) of a. c-e, Magnified views of the region containing the A570L and T572I mutations in the 'up' RBD protomer (c), the 'up'-coupled 'down' RBD protomer (d) and the 'down' RBD protomer (e) of the asymmetric 1-RBD up spike. The cryo-EM reconstruction is shown as a transparent surface with the underlying fitted model in cartoon representation and residues as balls and sticks. $\mathbf{f}-\mathbf{h}$, The 1-RBD up structure of the unmutated spike (PDB 6VYB) (shown in black and gray) superimposed on the u1S2q 1-RBD up structure (PDB 6X2B, this study), colored according to the coloring scheme in a. Overlays are shown for the 'up' RBD protomer (f), the 'up'-coupled 'down' RBD protomer (g) and the 'down' RBD protomer $(\mathbf{h})$.

intermediate SD1 configuration (Fig. 5e,h). The up state RBD in the $\mathrm{u} 1 \mathrm{~S} 2 \mathrm{q}$ construct resided largely in the position occupied in the unmutated construct (Fig. $5 \mathrm{c}, \mathrm{f}$ ). This indicated that the effect of the mutations was primarily isolated to the down state and suggested these mutations act to destabilize the down state rather than to stabilize the up state. These features were largely recapitulated in the u1S2q 2-RBD up state conformation, with subdomain 1 retaining the shift in the down state RBD (Supplementary Table 1). The structural details presented here indicate that, while locking the down state RBD into its unmutated position had little impact on the overall configuration of S1, altering the disposition of SD1 had wide-ranging impacts, consistent with the observed virus-to-virus differences in the geometric analysis described in Fig. 1.

We measured the binding of u1S2q with antibody CR3022 and ACE-2 (Fig. $6 c$ and Extended Data Fig. $7 a-c$ ) and observed a modest but reproducible increase in ACE-2 binding for u1S2q compared to the unmutated spike, consistent with the higher propensity of u1S2q to adopt RBD-up conformations (Extended Data Fig. 7b,c). The affinities of the CR3022 antibody to the unmutated spike or u1S2q were similar and also consistent with the previously published affinity of CR3022 for an RBD-only construct ${ }^{17}$ (Fig. 6c). On docking of the crystal structure of the CR3022 Fab in complex with the RBD (PDB 6YLA) ${ }^{17}$ onto the 1-up and 2-up structures of u1S2q, we found that the CR3022 constant domain clashed with the NTD and the adjacent RBD in both the 1-up conformation and the 2-up conformations (Fig. 6d,e). As previously suggested ${ }^{17}$, CR3022 binding is therefore likely to require a conformational change that rotates the RBD away from the central axis of the spike. In summary, none of the structures of the structural states of the spike determined thus far are compatible with CR3022 binding and our data show that CR3022 must induce or capture this potentially transient state in both the unmutated and u1S2q spikes. 
a

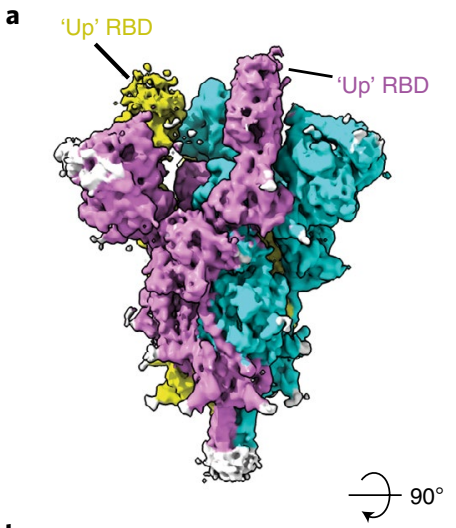

b

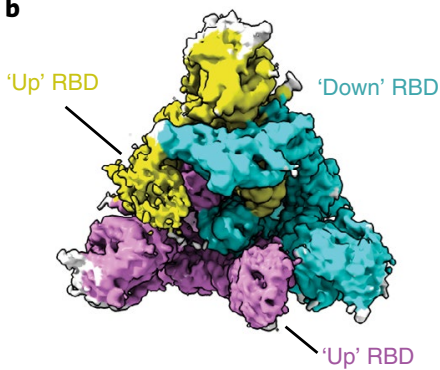

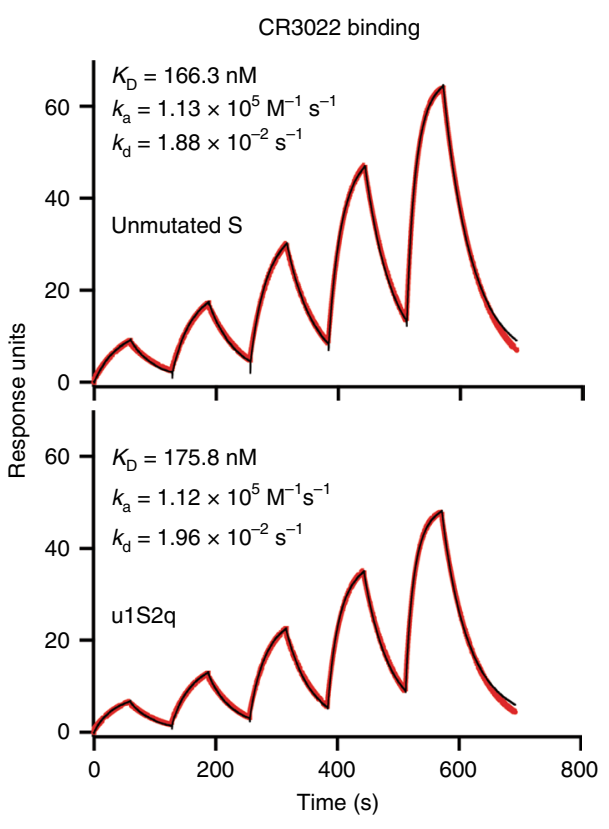

d CR3022 Fab docked on u1S2q 'up' state structures ‘1-RBD-up' structure ‘2-RBD-up’ structure
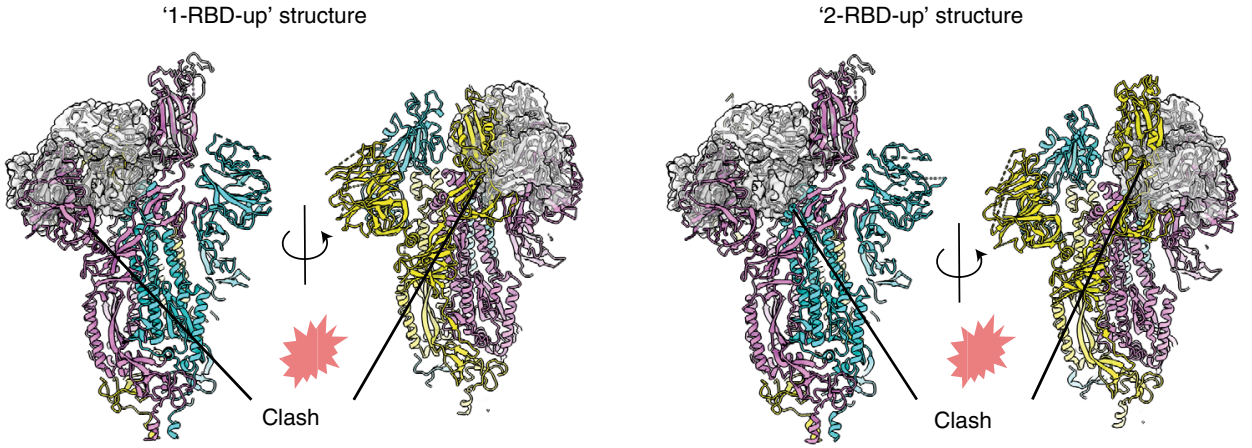

Fig. 6 | Structure of the u1S2q 2-RBD up state. a, Cryo-EM reconstruction of u1S2q, colored by chain. b, Rotated view (by $\sim 90^{\circ}$ ) of $\mathbf{a}$. c, Binding of antibody CR3022 to the unmutated construct (top) and u1s2q (bottom). Binding of CR3022 Fab to the S-proteins was measured by SPR using single-cycle kinetics. The black lines are the binding sensorgrams and the red lines show fits of the data to a 1:1 Langmuir binding model. d, CR3022 (shown as a semi-transparent, gray surface) modeled on the 1-up u1S2q structure (left) and the 2-up u1S2q structure (right) using RBD in the crystal structure of the CR3022-RBD complex (PDB 6W41) to superimpose on the up RBD of the u1S2q structures. Locations of potential clashes are indicated in each model.

\section{Discussion}

Conformational plasticity is a hallmark of the enveloped-virus fusion-protein structure due to the necessity of protecting the conserved viral fusion elements from host immune responses while retaining a sufficiently steep free-energy gradient to enable host cell fusion ${ }^{19}$. Exposed elements are generally well conditioned to be permissive and responsive to mutations through genetic drift and host immune adaptation. Conformational plasticity, however, presents an important difficulty in the context of vaccine and drug design. Indeed, lessons learned in the continued effort to produce a broadly protective HIV-1 vaccine have demonstrated the importance of a detailed understanding and control of fusion protein dynamics ${ }^{20-31}$. SARS-CoV-2 is probably no exception in this regard and, indeed, the conformational plasticity of the SARS-CoV-2 S-protein appears greater than that of the HIV-1 Env.

We aimed to develop a quantitative understanding of $\beta$-CoV structural states between viruses and within each RBD down and up state configuration. The wide breadth of domain arrangements and the relatively small contact area between the S1 and S2 subunits suggest that large changes in S-protein structure may occur from few mutations. A recent report indicated that the D614G mutation (located in the SD2 region and contacting S2) causes a potential fitness gain ${ }^{32}$. Based on our results showing that a disulfide linkage between SD2 and S2 in u2S2d decreased the population of up state RBDs (Extended Data Fig. 4b), the D614G mutation may indeed alter the conformational landscape of the SARS-CoV-2 S-protein. It was previously noted that the RBD up states observed in MERS and SARS-CoV-1 were not observed in the OC43, MHV or HKU1 S-protein structures ${ }^{10}$. We note that the SD1 u1S2q mutation sites are all asparagine in those other CoV S-proteins, and each displays a marked difference in SD1 positioning relative to the u1S2q structure. Thus, sequence differences at interdomain contact sites are not uncommon, and our observations suggest that the $\beta$-coronaviruses can quickly evade conformationally selective antibody responses. 
Table 1 | Cryo-EM data collection, refinement and validation statistics

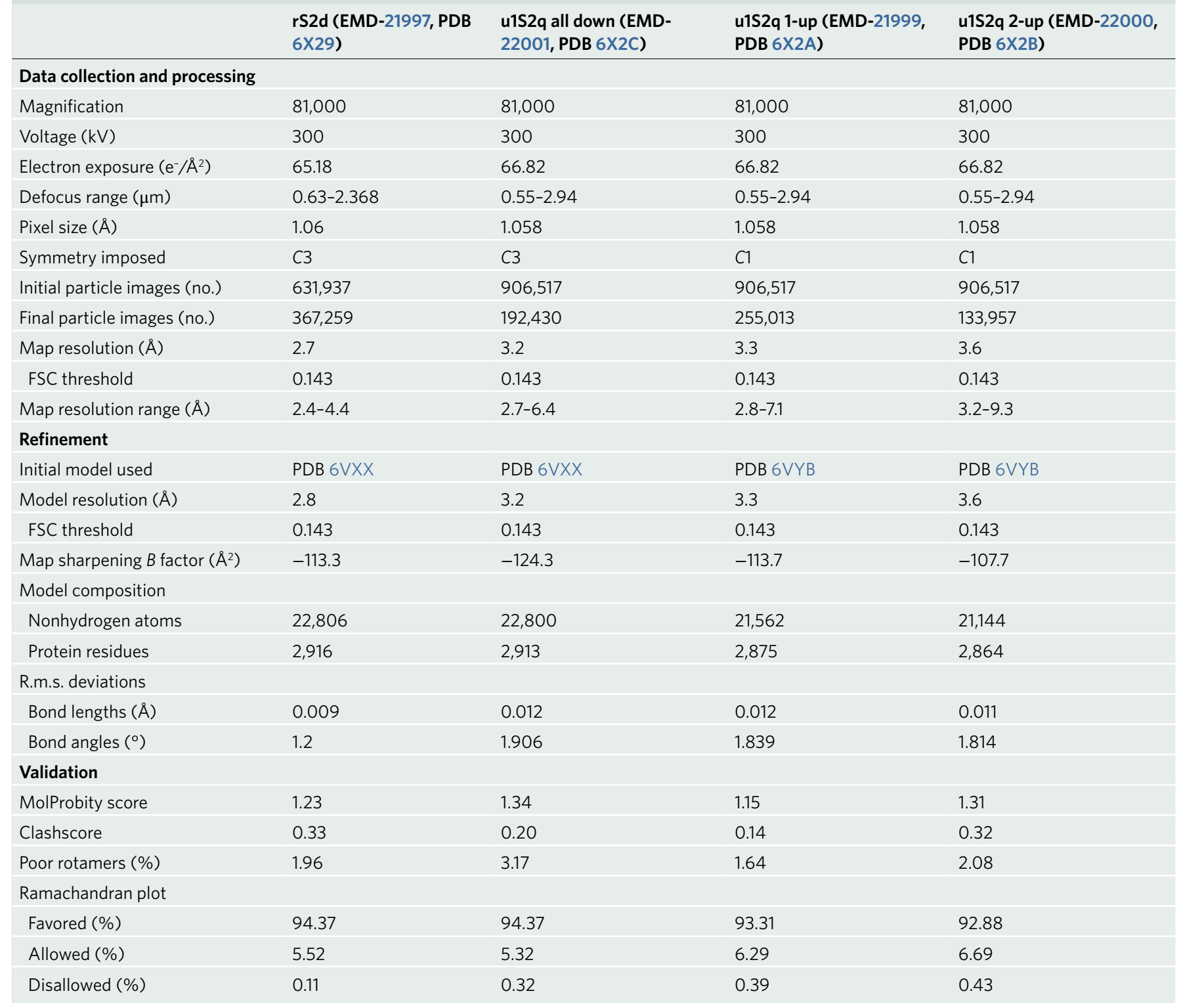

However, the degree to which this evasion mechanism is effectively utilized by the virus is uncertain, as such mutations may simultaneously incur a fitness penalty.

From the perspective of immunogen development, the constructs developed here present an opportunity to examine the ability of differentially stabilized S-protein particles to induce two different, yet important antibody responses. First, the u1S2q construct developed here displayed a prominent two-RBD up state distribution, demonstrating that interdomain contact modifications can lead to higher exposure of the immunogenic receptor-binding regions. Because RBD-directed responses that target the ACE- 2 binding site form a dominant proportion of neutralizing immune responses in infected patients $^{33}$, constructs that increase exposure and access to these sites could be useful candidates for vaccination regimens aimed at eliciting such responses. Indeed, full-length SARS-CoV-2 spike structures, either detergent-solubilized ${ }^{34}$ or virion-associated ${ }^{35}$, suggest that the down state is the predominant state that, in a vaccination context, may limit RBD-directed responses. The u1S2q mutations may therefore provide a means to enhance the induction of RBD responses in full-length S-protein immunogens. Furthermore, this design could also be used to identify receptor-binding-site-directed antibodies from patient sera, including potential new antibodies that may target sites that require a two-RBD up conformation. Second, the disulfide-linked down state locked double mutant $(\mathrm{rS} 2 \mathrm{~d})$ would presumably not elicit antibodies targeting the receptor binding site (these make up the majority of observed responses in convalescent patients ${ }^{36,37}$ ), but it would still be capable of eliciting antibodies such as S309 that are able to bind the down state $\mathrm{RBD}^{38}$. Indeed, a study of MERS responses suggests non-RBD responses (particularly NTD and S2 epitopes) will play an important role in vaccine-induced protection $^{39}$. From a theoretical perspective, the wide control over the $\mathrm{RBD}$ up/down distribution available to the virus suggests that, by analogy to HIV-1 viruses, which are notoriously difficult to neutralize, conformational blocking of antibody responses would not be unusual. Although there might be a fitness cost to the virus, it would not necessarily render the virion non-infectious. Using the double mutant $\mathrm{rS} 2 \mathrm{~d}$ as an immunogen provides a platform for inducing non-RBD responses that may be needed to protect against such evasion. Although the low expression yields of rS2d are a potential hindrance to its utility, introduction of other mutations, such as the 
recently described mutations designed to stabilize the S2 region ${ }^{40}$, may provide a way to further stabilize rS2d and increase its utility as an immunogen.

Complicating factors, such as a potential for antibody-induced enhancement, may favor the use of truncated, single-domain constructs with fewer potentially weak or non-neutralizing epitopes. Nevertheless, the designs presented here will allow for a detailed characterization of vaccine immunogenicity and antigenicity, paving the way for the development of vaccines for the novel SARS-CoV-2 and eventually a broadly protective $\beta-\mathrm{CoV}$ vaccine. Thus, although the previous generation of stabilizing mutations ensured well-folded spike trimers, the rational design approach developed here provides a means to controlling the RBD orientation distribution and should allow us to explore the impact of conformational dynamics from the perspective of vaccine and drug development.

\section{Online content}

Any methods, additional references, Nature Research reporting summaries, source data, extended data, supplementary information, acknowledgements, peer review information; details of author contributions and competing interests; and statements of data and code availability are available at https://doi.org/10.1038/ s41594-020-0479-4.

Received: 18 May 2020; Accepted: 7 July 2020;

Published online: 22 July 2020

\section{References}

1. Kirchdoerfer, R. N. et al. Pre-fusion structure of a human coronavirus spike protein. Nature 531, 118-121 (2016).

2. Hoffmann, M. et al. SARS-CoV-2 cell entry depends on ACE2 and TMPRSS2 and is blocked by a clinically proven protease inhibitor. Cell 181, 271-280 (2020).

3. Wrapp, D. et al. Cryo-EM structure of the 2019-nCoV spike in the prefusion conformation. Science 367, 1260-1263 (2020).

4. Yuan, Y. et al. Cryo-EM structures of MERS-CoV and SARS-CoV spike glycoproteins reveal the dynamic receptor binding domains. Nat. Commun. 8, 15092 (2017).

5. Gui, M. et al. Cryo-electron microscopy structures of the SARS-CoV spike glycoprotein reveal a prerequisite conformational state for receptor binding. Cell Res. 27, 119-129 (2017).

6. Song, W., Gui, M., Wang, X. \& Xiang, Y. Cryo-EM structure of the SARS coronavirus spike glycoprotein in complex with its host cell receptor ACE2. PLoS Pathog. 14, e1007236 (2018).

7. Kirchdoerfer, R. N. et al. Stabilized coronavirus spikes are resistant to conformational changes induced by receptor recognition or proteolysis. Sci. Rep. 8, 15701 (2018).

8. Walls, A. C. et al. Unexpected receptor functional mimicry elucidates activation of coronavirus fusion. Cell 176, 1026-1039 (2019).

9. Pallesen, J. et al. Immunogenicity and structures of a rationally designed prefusion MERS-CoV spike antigen. Proc. Natl Acad. Sci. USA 114, E7348-E7357 (2017).

10. Tortorici, M. A. et al. Structural basis for human coronavirus attachment to sialic acid receptors. Nat. Struct. Mol. Biol. 26, 481-489 (2019).

11. Walls, A. C. et al. Cryo-electron microscopy structure of a coronavirus spike glycoprotein trimer. Nature 531, 114-117 (2016).

12. Park, Y.-J. et al. Structures of MERS-CoV spike glycoprotein in complex with sialoside attachment receptors. Nat. Struct. Mol. Biol. 26, 1151-1157 (2019).

13. Walls, A. C. et al. Structure, function and antigenicity of the SARS-CoV-2 spike glycoprotein. Cell 180, 281-292 (2020).

14. Madhavi Sastry, G., Adzhigirey, M., Day, T., Annabhimoju, R. \& Sherman, W. Protein and ligand preparation: parameters, protocols and influence on virtual screening enrichments. J. Comput. Aided Mol. Des. 27, 221-234 (2013).

15. Salam, N. K., Adzhigirey, M., Sherman, W. \& Pearlman, D. A. Structure-based approach to the prediction of disulfide bonds in proteins. Protein Eng. Des. Sel. 27, 365-374 (2014).
16. Beard, H., Cholleti, A., Pearlman, D., Sherman, W. \& Loving, K. A. Applying physics-based scoring to calculate free energies of binding for single amino acid mutations in protein-protein complexes. PLoS ONE 8, e82849 (2013).

17. Yuan, M. et al. A highly conserved cryptic epitope in the receptor binding domains of SARS-CoV-2 and SARS-CoV. Science 368, 630-633 (2020).

18. ter Meulen, J. et al. Human monoclonal antibody combination against SARS coronavirus: synergy and coverage of escape mutants. PLoS Med. 3, e237 (2006)

19. Rey, F. A. \& Lok, S.-M. Common features of enveloped viruses and implications for immunogen design for next-generation vaccines. Cell 172, 1319-1334 (2018).

20. de Taeye, S. W. et al. Immunogenicity of stabilized HIV-1 envelope trimers with reduced exposure of non-neutralizing epitopes. Cell 163, 1702-1715 (2015).

21. He, L. et al. HIV-1 vaccine design through minimizing envelope metastability. Sci. Adv. 4, eaau6769 (2018).

22. Zhang, P. et al. Interdomain stabilization impairs CD4 binding and improves immunogenicity of the HIV-1 envelope trimer. Cell Host Microbe 23, 832-844 (2018).

23. Chuang, G.-Y. et al. Structure-based design of a soluble prefusion-closed HIV-1 Env trimer with reduced CD4 affinity and improved immunogenicity. J. Virol. 91, e02268-16 (2017).

24. Torrents de la Peña, A. et al. Improving the immunogenicity of nativelike HIV-1 envelope trimers by hyperstabilization. Cell Rep. 20, 1805-1817 (2017).

25. Medina-Ramírez, M. et al. Design and crystal structure of a native-like HIV-1 envelope trimer that engages multiple broadly neutralizing antibody precursors in vivo. J. Exp. Med. 214, 2573-2590 (2017).

26. Steichen, J. M. et al. HIV vaccine design to target germline precursors of glycan-dependent broadly neutralizing antibodies. Immunity $\mathbf{4 5}$, 483-496 (2016)

27. Kulp, D. W. et al. Structure-based design of native-like HIV-1 envelope trimers to silence non-neutralizing epitopes and eliminate CD4 binding. Nat. Commun. 8, 1655 (2017).

28. Yang, L. et al. Structure-guided redesign improves NFL HIV Env trimer integrity and identifies an inter-protomer disulfide permitting post-expression cleavage. Front. Immunol. 9, 1631 (2018).

29. Sharma, S. K. et al. Cleavage-independent HIV-1 Env trimers engineered as soluble native spike mimetics for vaccine design. Cell Rep. 11, 539-550 (2015)

30. Guenaga, J. et al. Structure-guided redesign increases the propensity of HIV Env to generate highly stable soluble trimers. J. Virol. 90, 2806-2817 (2016).

31. Sliepen, K. et al. Structure and immunogenicity of a stabilized HIV-1 envelope trimer based on a group-M consensus sequence. Nat. Commun. 10, 2355 (2019).

32. Korber, B. et al. Tracking changes in SARS-CoV-2 Spike: evidence that D614G increases infectivity of the COVID-19 virus. Cell https://doi. org/10.1016/j.cell.2020.06.043 (2020).

33. Barnes, C. O. et al. Structures of human antibodies bound to SARS-CoV-2 spike reveal common epitopes and recurrent features of antibodies. Cell 182, $1-15$ (2020).

34. Cai, Y. et al. Distinct conformational states of SARS-CoV-2 spike protein Preprint at bioRxiv https://doi.org/10.1101/2020.05.16.099317 (2020).

35. Turoňová, B. et al. In situ structural analysis of SARS-CoV-2 spike reveals flexibility mediated by three hinges. Preprint at bioRxiv https://doi. org/10.1101/2020.06.26.173476 (2020)

36. Zost, S. J. et al. Rapid isolation and profiling of a diverse panel of human monoclonal antibodies targeting the SARS-CoV-2 spike protein. Nat. Med. https://doi.org/10.1038/s41591-020-0998-x (2020).

37. Brouwer, P. J. M. et al. Potent neutralizing antibodies from COVID-19 patients define multiple targets of vulnerability. Science https://doi. org/10.1126/science.abc5902 (2020).

38. Pinto, D. et al. Cross-neutralization of SARS-CoV-2 by a human monoclonal SARS-CoV antibody. Nature 583, 290-295 (2020).

39. Wang, L. et al. Importance of neutralizing monoclonal antibodies targeting multiple antigenic sites on the Middle East Respiratory Syndrome coronavirus spike glycoprotein to avoid neutralization escape. J. Virol. 92, e02002-e02017 (2018)

40. Hsieh, C.-L. et al. Structure-based design of prefusion-stabilized SARS-CoV-2 spikes. Preprint at bioRxiv https://doi.org/10.1101/2020.05.30.125484 (2020).

Publisher's note Springer Nature remains neutral with regard to jurisdictional claims in published maps and institutional affiliations.

(C) The Author(s), under exclusive licence to Springer Nature America, Inc. 2020 


\section{Methods}

Vector-based analysis. Vector analysis was performed using available cryo-EM structures for SARS-CoV-2 ${ }^{3,13}, \mathrm{SARS}^{4,5,7,8}, \mathrm{MERS}^{4,9}$ and other human ${ }^{1,10}$ and murine $^{11} \beta-\mathrm{CoV}$ spike proteins. Domains for the vector analysis were selected based on visual inspection of alignments between SARS, MERS and SARS-CoV-2 structures. Specifically, C $\alpha$ centroids were determined for the S1 NTD, RBD, SD1 and SD2 (SARS-CoV-2 residues 27-43 and 54-271, 330-443 and 503-528, 323-329 and 529-590, 294-322 and 591-696, respectively; equivalent SARS/ MERS/murine/HKU1/OC43 residues selected based on structural alignment with SARS-CoV-2), as well as a $\beta$-sheet motif in the NTD (residues 116-129 and 169-172) and a helix motif in the RBD (residues 403-410). The NTD was split into two regions with the SD1-contacting, SD2-adjacent portion referred to here as NTD' (residues 44-53 and 272-293). C $\alpha$ centroids in the S2 subunit were obtained for a $\beta$-sheet motif (residues 717-727 and 1047-1071) and the CD domain (711-716 and 1072-1122). Vector magnitudes, angles and dihedrals between these centroids were determined and used in the subsequent analysis. Vector analysis was performed using the $\mathrm{VMD}^{41} \mathrm{Tcl}$ interface. PCA was performed in $\mathrm{R}$ with the vector data centered and scaled ${ }^{42}$

Rational structure-based design. Structures for SARS-COV-1 (PDB 5X584), MERS (PDB 6Q04 ${ }^{12}$ ) and SARS-CoV-2 (PDB 6VXX ${ }^{13}$ ) were prepared in Maestro ${ }^{43}$ using the protein preparation wizard ${ }^{14}$ followed by in silico mutagenesis using Schrödinger's cysteine mutation ${ }^{15}$ and residue scanning ${ }^{16}$ tools. Residue scanning was first performed for individual selected sites allowing mutations to Leu, Ile, Trp, Tyr and Val, followed by scanning of combinations for those that yielded a negative overall score. Scores and visual inspection were used in the selection of the prepared constructs.

Protein expression and purification. The SARS-CoV-2 ectodomain constructs were produced and purified as described previously ${ }^{3}$. Briefly, a gene encoding residues 1-1208 of the SARS-CoV-2 S (GenBank MN908947) with proline substitutions at residues 986 and 987, a 'GSAS' substitution at the furin cleavage site (residues 682-685), a C-terminal T4 fibritin trimerization motif, an HRV3C protease cleavage site, a TwinStrepTag and an 8XHisTag was synthesized and cloned into the mammalian expression vector $\mathrm{p} \alpha \mathrm{H}$. All mutants were introduced in this background. Expression plasmids encoding the ectodomain sequence were used to transiently transfect FreeStyle293F cells using Turbo293 (SpeedBiosystems). Protein was purified on the sixth day post-transfection from the filtered supernatant using StrepTactin resin (IBA).

Antibody CR3022 was produced in Expi293 cells and purified by Protein A affinity. To express the ACE- 2 constructs, the ACE- 2 gene was cloned as a fusion protein with either the human or mouse $\mathrm{Fc}$ region attached to the $\mathrm{C}$-terminal end of ACE-2. A 6X His-tag was added to the C-terminal end of the Fc domain of each construct. ACE-2 with human Fc tag was purified by Protein A affinity chromatography, and ACE-2 with mouse Fc tag was purified by Ni-NTA chromatography.

Thermal shift assay. The thermal shift assay was performed using Tycho NT.6 (NanoTemper Technologies). Spike variants were diluted $\left(0.15 \mathrm{mg} \mathrm{ml}^{-1}\right)$ in nCoV buffer ( $2 \mathrm{mM}$ Tris, $\mathrm{pH} 8.0,200 \mathrm{mM} \mathrm{NaCl}, 0.02 \%$ sodium azide) and run in duplicates in capillary tubes. Intrinsic fluorescence was recorded at $330 \mathrm{~nm}$ and $350 \mathrm{~nm}$ while heating the sample from 35 to $95^{\circ} \mathrm{C}$ at a rate of $3{ }^{\circ} \mathrm{C} \mathrm{min}{ }^{-1}$. The ratio of fluorescence $(350 / 330 \mathrm{~nm})$ and the inflection temperatures (Ti) were calculated by Tycho NT.6.

Negative-stain electron microscopy. A $100 \mu \mathrm{g} \mathrm{ml}^{-1}$ final concentration of the spike was made in $5 \%$ glycerol in HBS pH $7.4(20 \mathrm{mM}$ HEPES, $150 \mathrm{mM} \mathrm{NaCl})$ with 7.5 $\mathrm{mM}$ gluteraldehyde at room temperature. After 5-min incubation, gluteraldehyde was quenched by adding sufficient $1 \mathrm{M}$ Tris stock, $\mathrm{pH} 7.4$ to give a final concentration of $75 \mathrm{mM}$ Tris and incubated for $5 \mathrm{~min}$. The carbon-coated grids (CF300-cu, EMS) were glow-discharged for $20 \mathrm{~s}$ at $15 \mathrm{~mA}$. A 5- $\mu$ l sample incubated on grid for $10-15 \mathrm{~s}$ was blotted and then stained with $2 \%$ uranyl formate. Images were obtained with a Philips EM420 electron microscope operated at $120 \mathrm{kV}$, at $\times 82,000$ magnification and with a pixel size of $4.02 \AA$. The RELION ${ }^{44}$ program was used to perform class averaging of the single-particle images.

Cryo-electron microscopy sample preparation, data collection and processing. Purified SARS-CoV-2 spike preparations were diluted to a concentration of $\sim 1 \mathrm{mg} \mathrm{ml}^{-1}$ in $2 \mathrm{mM}$ Tris $\mathrm{pH} 8.0,200 \mathrm{mM} \mathrm{NaCl}$ and $0.02 \% \mathrm{NaN}_{3}$, then $2.5 \mu \mathrm{l}$ of protein was deposited on a CF-1.2/1.3 grid that had been glow-discharged for $30 \mathrm{~s}$ in a PELCO easiGlow glow discharge cleaning system. After 30-s incubation in $>95 \%$ humidity, excess protein was blotted away for $2.5 \mathrm{~s}$ before being plunge-frozen into liquid ethane using a Leica EM GP2 plunge freezer (Leica Microsystems). Frozen grids were imaged in a Titan Krios system (Thermo Fisher) equipped with a K3 detector (Gatan). Data were acquired using the Leginon system ${ }^{45}$. The dose was fractionated over 50 raw frames and collected at a 50-ms frame rate. This dataset was energy-filtered with a slit width of $30 \mathrm{eV}$. Individual frames were aligned and dose-weighted ${ }^{46}$. CTF estimation, particle picking, 2D classifications, ab initio model generation, heterogeneous refinements, homogeneous 3D refinements and local resolution calculations were carried out in cryoSPARC $^{47}$.

Cryo-electron microscopy structure fitting and analysis. Structures of the all down state (PDB 6VXX) and single RBD up state (PDB 6VYB) from the previously published SARS-CoV-2 ectodomain were used to fit the cryo-EM maps in Chimera ${ }^{48}$. The 2-RBD up state was generated in PyMol using the single RBD up state structure. Mutations were made in $\mathrm{PyMol}^{49}$. Coordinates were then fitted manually in $\operatorname{Coot}^{50}$ followed by iterative refinement using Phenix ${ }^{51}$ real space refinement and subsequent manual coordinate fitting in Coot. Structure and map analysis were performed using PyMol, Chimera ${ }^{48}$ and ChimeraX ${ }^{52}$.

Surface plasmon resonance. The binding of antibody CR3022 and ACE- 2 to the SARS-CoV-2 spike constructs was assessed by SPR, in two experimental formats, on a Biacore T-200 system (GE Healthcare) at $25^{\circ} \mathrm{C}$ using HBS-EP+ $(10 \mathrm{mM}$ HEPES, pH 7.4, $150 \mathrm{mM} \mathrm{NaCl}, 3 \mathrm{mM}$ EDTA and $0.05 \%$ surfactant $\mathrm{P}-20$ ) as the running buffer. In the first format, the spike constructs were captured on an SA chip and were assayed by flowing over CR3022 IgG or ACE-2-Fc. The surface was regenerated between injections by flowing over SA regeneration buffer $(1 \mathrm{M} \mathrm{NaCl}$, $50 \mathrm{mM} \mathrm{NaOH}$ ) solution for $10 \mathrm{~s}$ with a flow rate of $100 \mu \mathrm{lmin}^{-1}$. Blank sensorgrams were obtained by injection of the same volume of HBS-EP+ buffer in place of IgGs and Fab solutions. Sensorgrams were corrected with corresponding blank curves. In the second format, CR3022 IgG or ACE-2-human Fc was captured on CM5 chip immobilized with human Anti-Fc (8,000 RU), and binding was measured by flowing over each of the spike constructs at $200 \mathrm{nM}$ in running buffer. The surface was regenerated between injections by flowing over $3 \mathrm{M} \mathrm{MgCl}_{2}$ solution for $10 \mathrm{~s}$ with a flow rate of $100 \mu \mathrm{min}^{-1}$. To assess the affinity of CR3022 to the unmutated and $\mathrm{u} 1 \mathrm{~S} 2 \mathrm{q}$ spikes, the spikes were captured on an SA chip as described above, and five concentrations of CR3022 were injected in a single-cycle kinetics format. Sensorgram data were analyzed using the BiaEvaluation software (GE Healthcare).

Reporting Summary. Further information on research design is available in the Nature Research Reporting Summary linked to this Article.

\section{Data availability}

Cryo-EM reconstructions and atomic models have been deposited in the EMDB and wwPDB with accession codes EMD-21997, EMD-21999, EMD-22000 and EMD-22001 and PDB 6X29, 6X2A, 6X2B and 6X2C. Source data for graphs are available in Supplementary Data 1.

\section{Code availability}

The code developed to determine vector magnitudes, angles and dihedrals for this study is available from the corresponding authors upon reasonable request.

\section{References}

41. Humphrey, W., Dalke, A. \& Schulten, K. VMD: visual molecular dynamics. J. Mol. Graph. 14, 33-38 (1996).

42. R Core Team R: A Language and Environment for Statistical Computing (R Foundation for Statistical Computing, 2017).

43. Maestro (Schrödinger, 2020).

44. Scheres, S. H. W. in Methods in Enzymology Vol. 579 (ed. Crowther, R. A.) 125-157 (Academic Press, 2016).

45. Suloway, C. et al. Automated molecular microscopy: the new Leginon system. J. Struct. Biol. 151, 41-60 (2005).

46. Zheng, S. Q. et al. MotionCor2: anisotropic correction of beam-induced motion for improved cryo-electron microscopy. Nat. Methods 14, 331-332 (2017).

47. Punjani, A., Rubinstein, J. L., Fleet, D. J. \& Brubaker, M. A. cryoSPARC: algorithms for rapid unsupervised cryo-EM structure determination. Nat. Methods 14, 290-296 (2017).

48. Pettersen, E. F. et al. UCSF Chimera-a visualization system for exploratory research and analysis. J. Comput. Chem. 25, 1605-1612 (2004).

49. The PyMOL Molecular Graphics System (Schrödinger, 2015).

50. Emsley, P., Lohkamp, B., Scott, W. G. \& Cowtan, K. Features and development of Coot. Acta Crystallogr. D Biol. Crystallogr. 66, 486-501 (2010).

51. Afonine, P. V. et al. Real-space refinement in PHENIX for cryo-EM and crystallography. Acta Crystallogr. D Struct. Biol. 74, 531-544 (2018).

52. Goddard, T. D. et al. UCSF ChimeraX: meeting modern challenges in visualization and analysis. Protein Sci. 27, 14-25 (2018).

\section{Acknowledgements}

Initial cryo-EM data were collected on the Titan Krios system at the Shared Materials and Instrumentation Facility in Duke University. Data collection for high-resolution structure determination was performed at the National Center for Cryo-EM Access and Training (NCCAT) and the Simons Electron Microscopy Center located at the New York Structural Biology Center, supported by the NIH Common Fund Transformative High Resolution Cryo-Electron Microscopy program (U24 GM129539) and by grants 
from the Simons Foundation (SF349247) and NY State. We thank E. Eng, D. Bobe, M. Walters and $\mathrm{H}$. Leddy for microscope alignments and assistance with cryo-EM data collection as well as J. McLellan for providing the plasmid for the unmutated construct and K. Manne for preparing the CR3022 Fab. This work was supported by U19

AI142596 (B.F.H.), Integrated Preclinical/Clinical AIDS Vaccine Development Program, NIAID, NIH; Duke University Center for AIDS Research (CFAR); an administrative supplement to NIH R01 AI145687 for coronavirus research and Translating Duke Health Initiative (P.A. and R.C.H.), the Intramural Research Program of the NIH; National Institute of Environmental Health Sciences (ZIC ES103326 to M.J.B.), and a contract from the State of North Carolina Pandemic Recovery Office through funds from the Coronavirus Aid, Relief and Economic Security (CARES) Act. This study utilized the computational resources offered by Duke Research Computing (http://rc.duke.edu; NIH 1S10OD018164-01) at Duke University. We thank M. DeLong, C. Kneifel, M. Newton, V. Orlikowski, T. Milledge and D. Lane from the Duke Office of Information Technology and Research Computing for providing assistance with setting up and maintaining the computing environment.

\section{Author contributions}

R.H. and P.A. conceived the study, determined cryo-EM structures and analyzed data. R.H. led computational studies. P.A. led structural studies. R.J.E. led NSEM studies and analyzed data. K.M. collected NSEM data and performed initial data analysis. K.J., V.S., S.G. and D.L. produced, purified and analyzed proteins. M.K. and A.L.H. optimized cryo-EM specimens. K.J. and R.P. performed binding studies to optimize spike preparations. R.J.E., M.J.B., B.F.H. and P.A. supervised studies. R.H. and P.A. wrote the manuscript with help from all authors.

\section{Competing interests}

The authors declare no competing interests.

\section{Additional information}

Extended data is available for this paper at https://doi.org/10.1038/s41594-020-0479-4. Supplementary information is available for this paper at https://doi.org/10.1038/ s41594-020-0479-4.

Correspondence and requests for materials should be addressed to R.H. or P.A. Peer review information Inês Chen was the primary editor on this article and managed its editorial process and peer review in collaboration with the rest of the editorial team.

Reprints and permissions information is available at www.nature.com/reprints. 

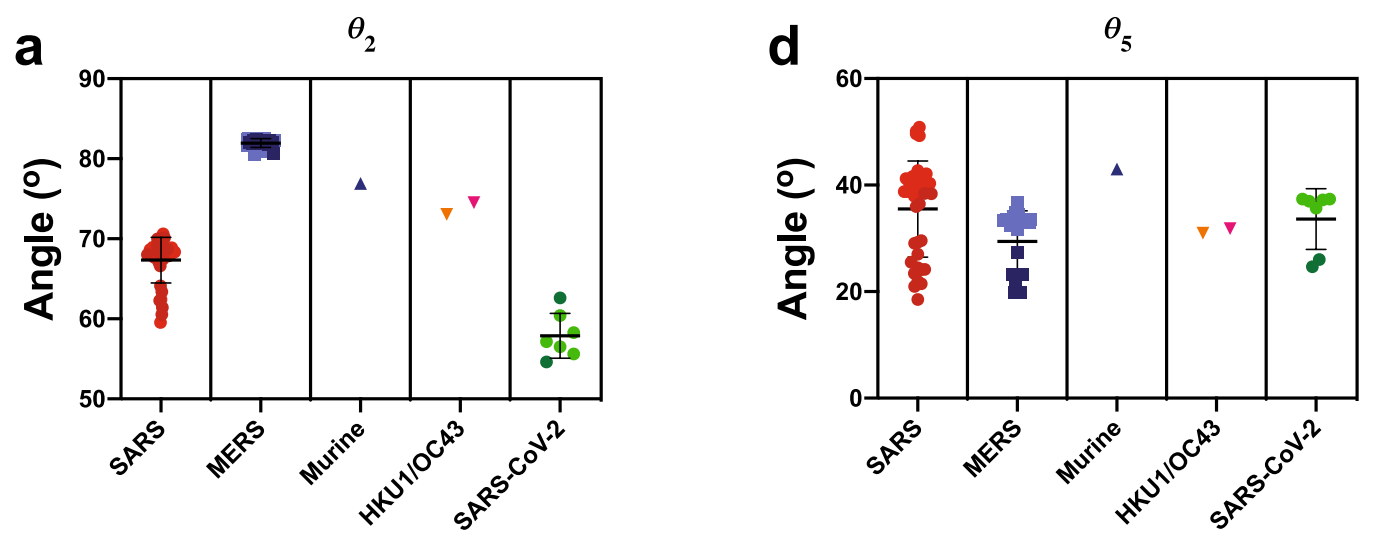

b
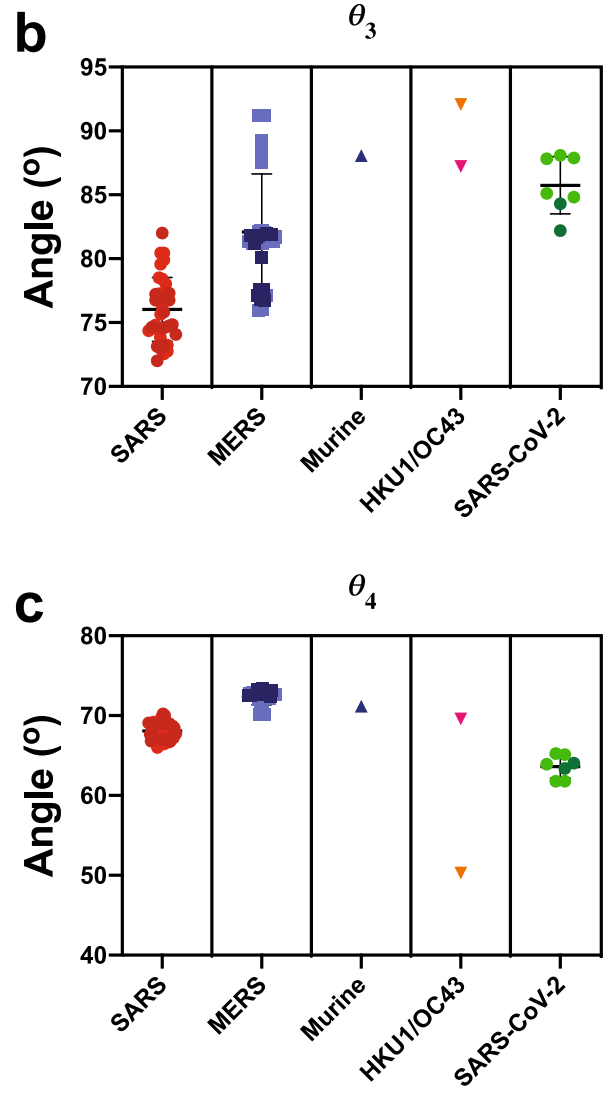
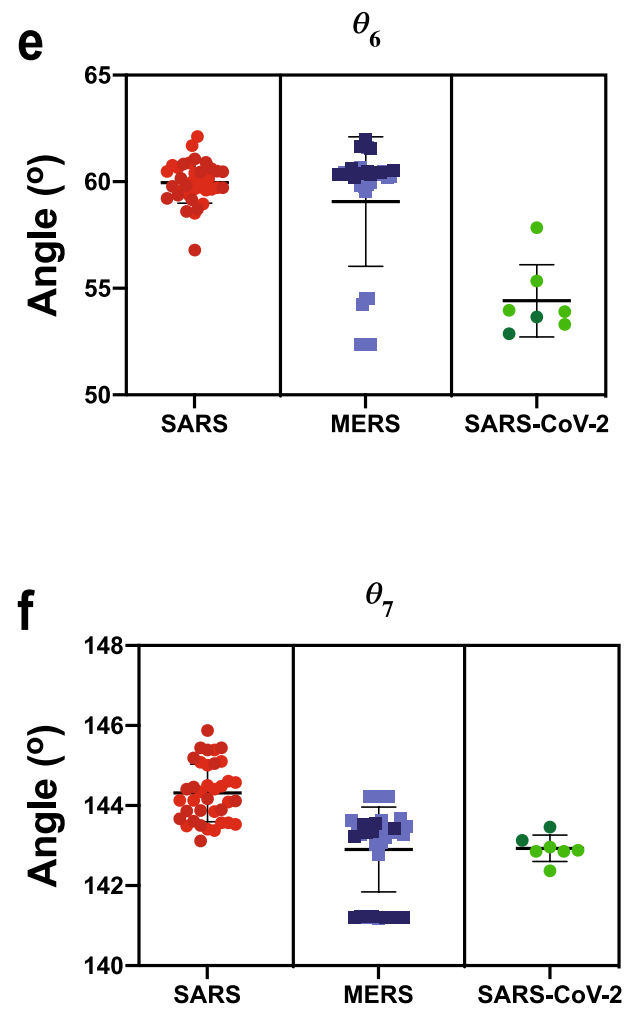

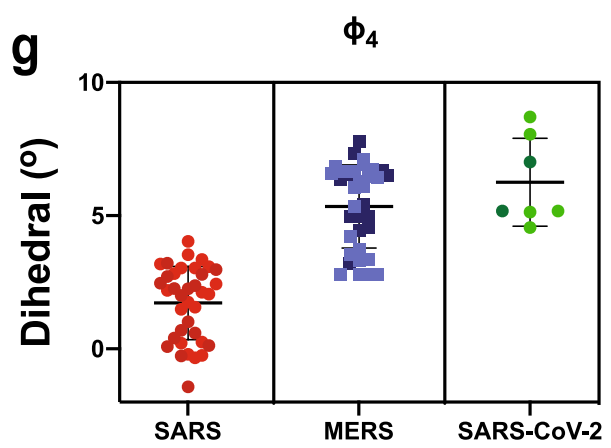

Extended Data Fig. 1 | See next page for caption. 
Extended Data Fig. 1 | $\beta$-CoV Vector Analysis. a, The angle between the vectors connecting the NTD sheet motif centroid and the NTD centroid and the vector connecting the the NTD centroid to the NTD' centroid. $\mathbf{b}$, The angle between the vectors connecting the NTD' centroid and the SD2 centroid and the vector connecting the the SD2 centroid to the SD1 centroid. c, The angle between the vectors connecting the NTD centroid and the NTD' centroid and the vector connecting the the NTD' centroid to the SD2 centroid. d, The angle between the vectors connecting the SD1 centroid and the RBD centroid and the vector connecting the the RBD centroid to the RBD helix motif centroid. e, The angle between the vectors connecting the NTD' centroid and the SD2 centroid and the vector connecting the the SD2 centroid to the CD centroid. f, The angle between the vectors connecting the SD2 centroid and the CD centroid and the vector connecting the the CD centroid to the $\beta$-sheet motif centroid. $\mathbf{g}$, The dihedral about the SD2 centroid and the CD centroid. Points for SARS, MERS, and SARS-2 in Fig. 1 (g)-(j) colored according to 'up' (dark) and 'down' (light) states according to the color code in the PCA analysis, panels $(\mathbf{e})$ and $(\mathbf{f})$. Individual data points shown as symbols; lines denote mean and s.d. The source data are available in Supplementary Data 1. 
RBD to S2

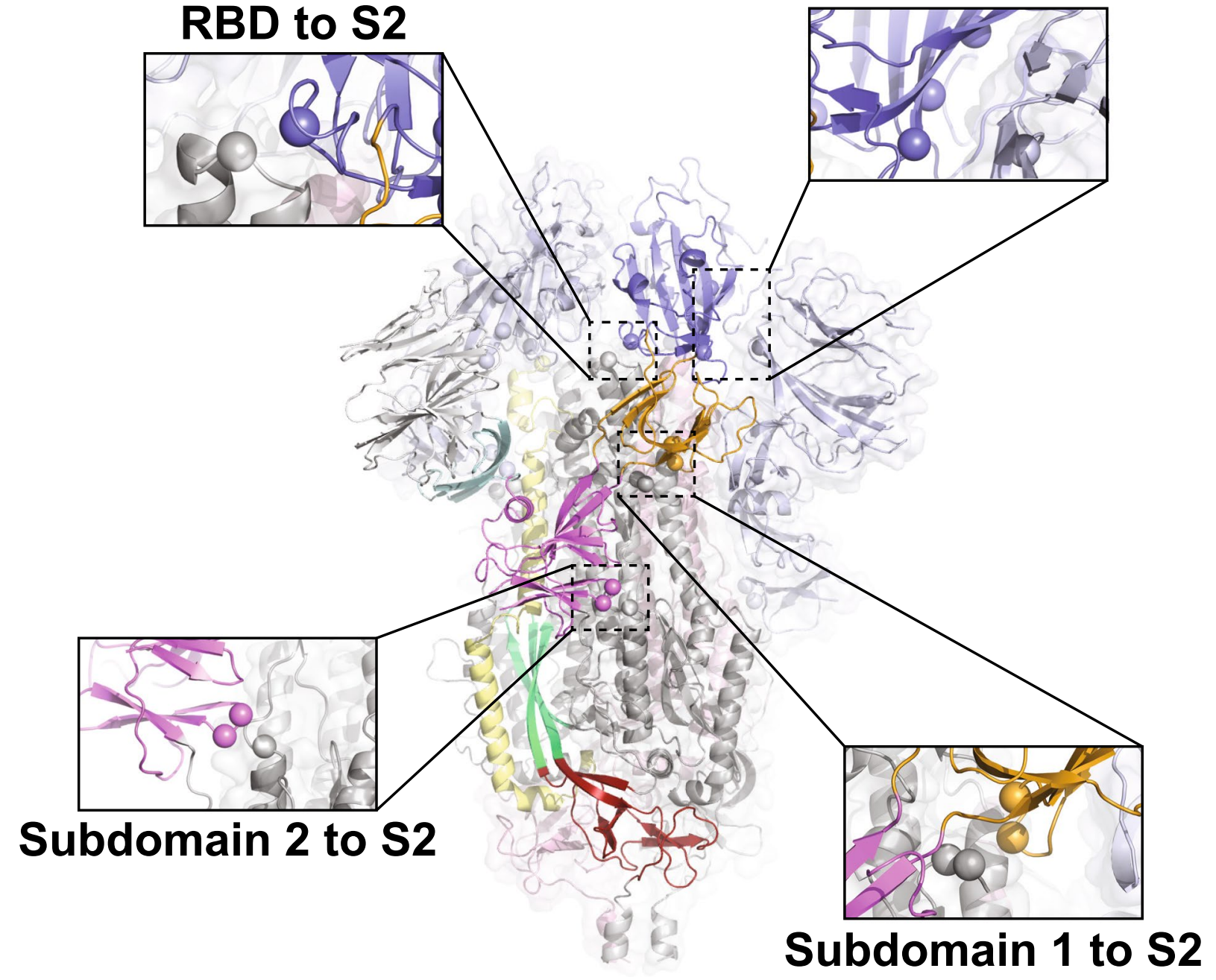

Extended Data Fig. 2 | Sites identified for differential stabilization of the SARS-CoV-2 S-protein. Single protomer colored according to Fig. 1 with remaining two protomers color according to S1 (light blue) and S2 (gray). Spheres indicate candidate mutation sites. 
a

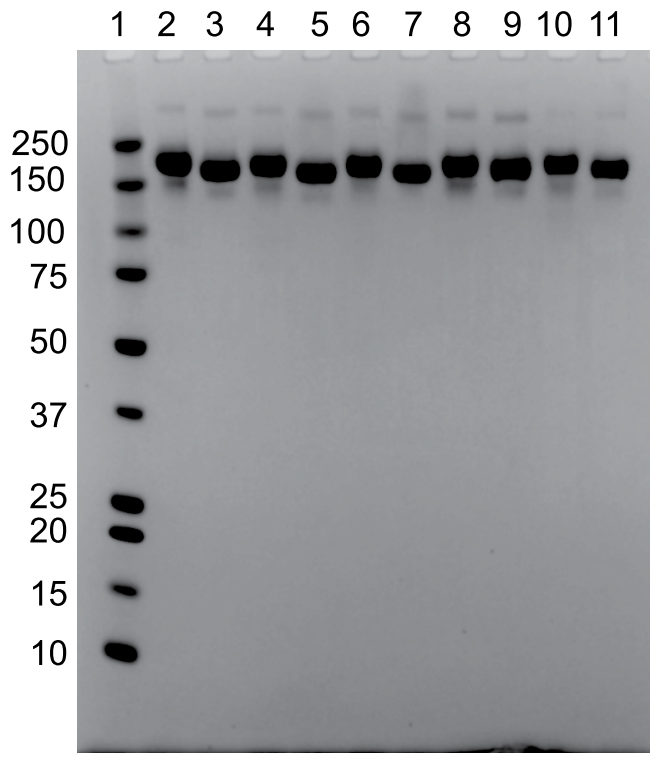

$\begin{array}{lll}12 & 13 & 14\end{array}$

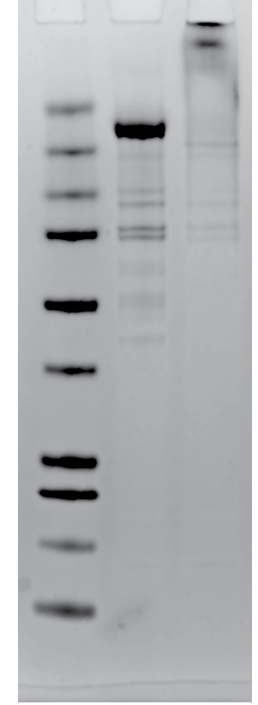

Legend:

1. Marker

2. Reduced Unmutated S

3. Non-Reduced Unmutated S

4. Reduced u2S2d

5. Non-Reduced u2S2d

6. Reduced $\mathrm{rNt}$

7. Non-Reduced rNt

8. Reduced u1S2q

9. Non-Reduced u1S2q

10. Reduced u2S2d

11. Non-Reduced u1S2d

12. Marker

13. Reduced rS2d

14. Non-reduced rS2d

b

\begin{tabular}{|c|c|}
\hline Spike construct & Yield (mg) \\
\hline Unmutated & $3-5$ \\
\hline u1S2q & 2.15 \\
\hline rs2d & 0.168 \\
\hline u1S2d & 0.65 \\
\hline u2S2d & 0.3 \\
\hline rNt & 0.26 \\
\hline
\end{tabular}

Extended Data Fig. 3 | SDS-PAGE and yields of purified S protein constructs. a, SDS-PAGE gels of the S protein constructs. b, Yields/L of the S protein constructs. 
a

\begin{tabular}{r|ll|} 
Construct: & rNt \\
Mutations: & D389L, S514L, E516L \\
Class: & 3-Down & 1-Up \\
\# Particles: & 39,128 & 43,398 \\
$\%$ Tota: $:$ & $47 \%$ & $53 \%$ \\
Resolution (A): & 18.4 & 18.4 \\
\cline { 2 - 2 }
\end{tabular}
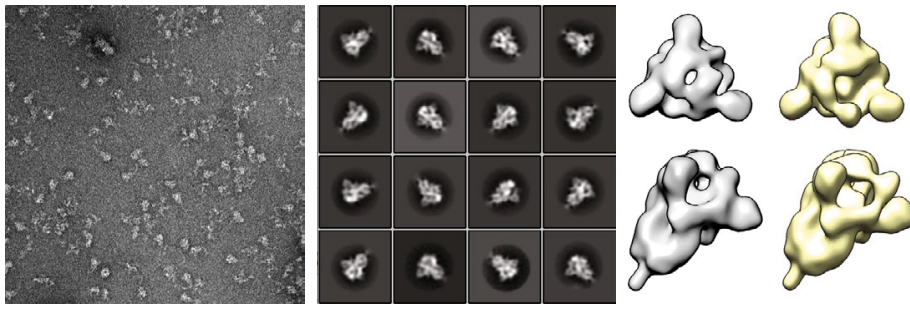

b

\begin{tabular}{r|ll|}
\cline { 2 - 3 } Construct: & u2S2d \\
Mutations: & \multicolumn{2}{|l|}{ G669C, } \\
\cline { 2 - 3 } Clas66C \\
\cline { 2 - 3 } 3-Down & 1-Up \\
\# Particles: & 89,842 & 42,060 \\
$\%$ Total: & $68 \%$ & $32 \%$ \\
solution (A): & 17.5 & 18.4 \\
\cline { 2 - 2 } & &
\end{tabular}

C
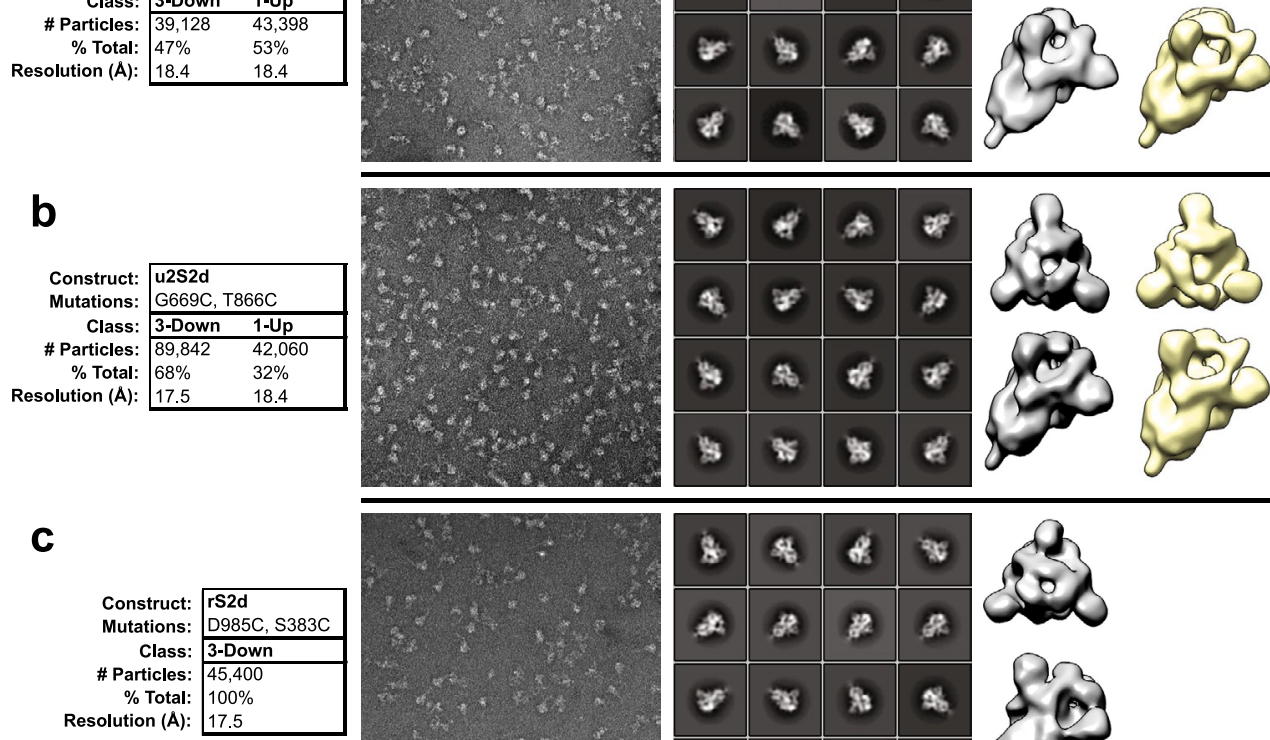

Resolution (A): 17.5
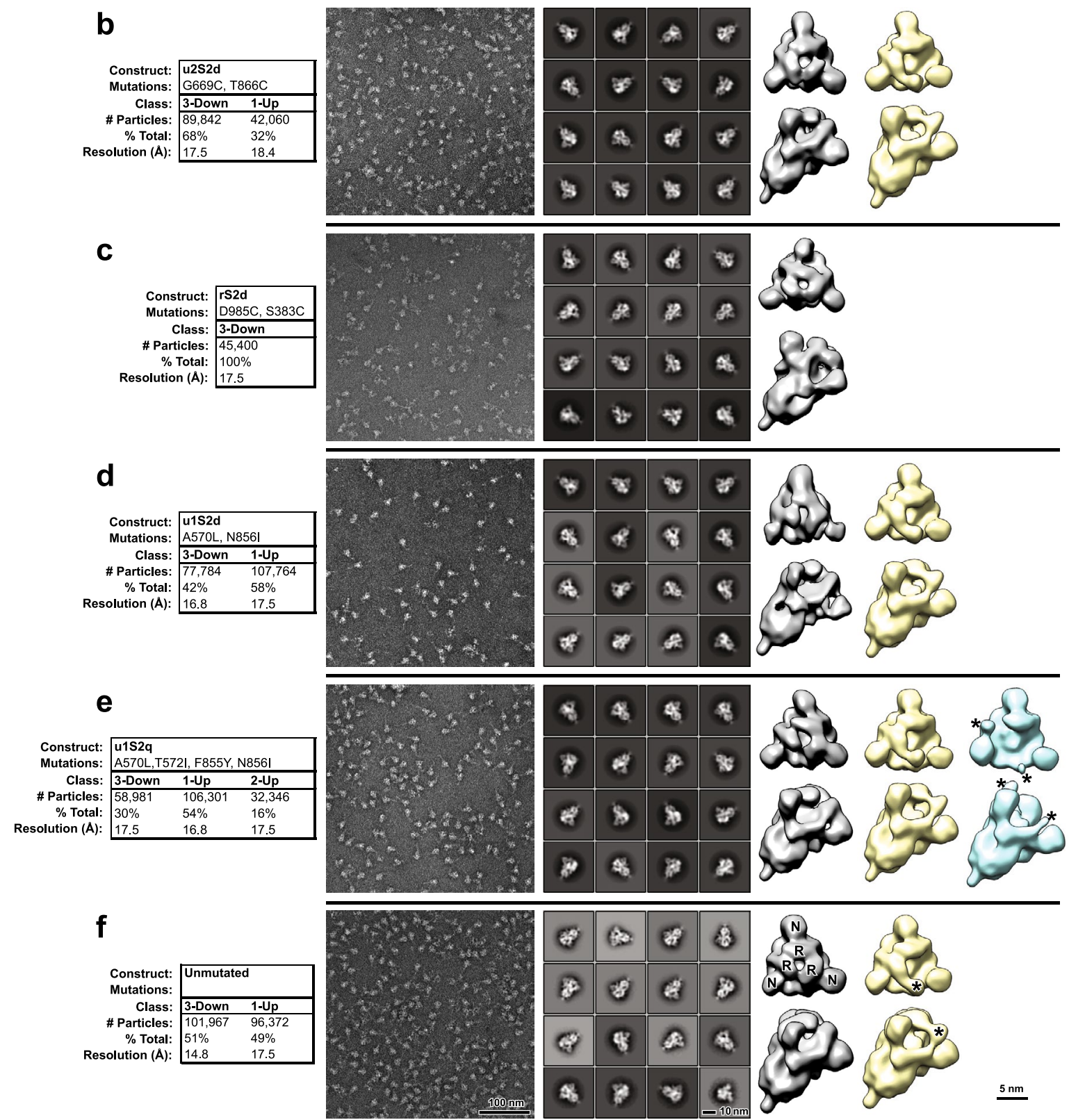

Extended Data Fig. 4 | Negative stain electron microscopy analysis of S-protein constructs. a, Data tables, indicating construct names, mutations, observed classes, number and percent of particles per class and final resolution (gold-standard Fourier-shell correlation, 0.143 level). b, Raw micrographs. c, Representative 2D class averages. d, 3D reconstructions of 3-RBD-down classes, shown in top view, looking down the S-protein 3-fold axis on the left and tilted view on the right. Receptor binding domains and $\mathrm{N}$-terminal domains of first structure marked with $\mathrm{R}$ and $\mathrm{N}$, respectively. $\mathbf{e}, 3 \mathrm{D}$ reconstructions of 1-RBD-up classes. Up-RBD is marked with an asterisk. $\mathbf{f}$, 3D reconstruction of 2-RBD-up class. Density for up-RBDs is weak, indicated by asterisks. 
a

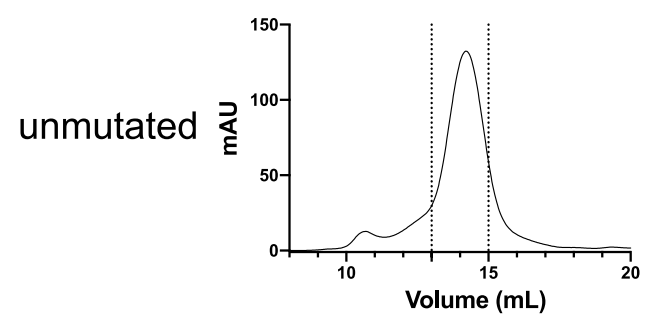

b

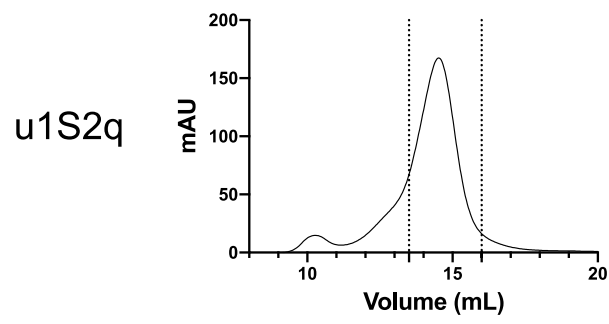

C

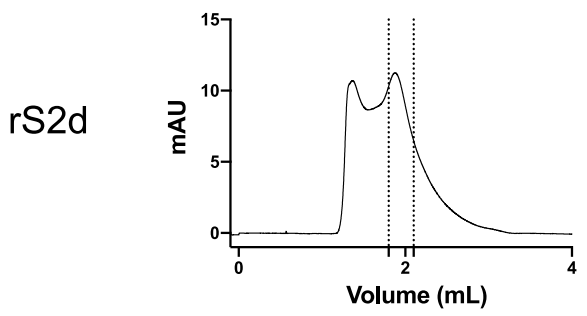

d

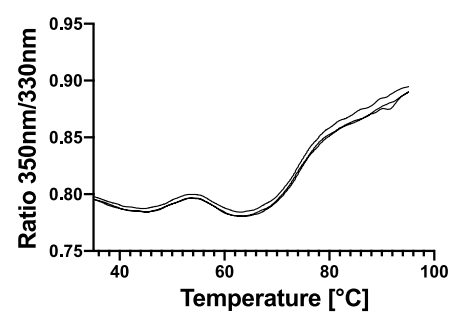

e

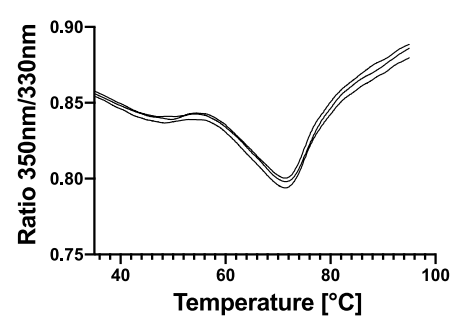

f

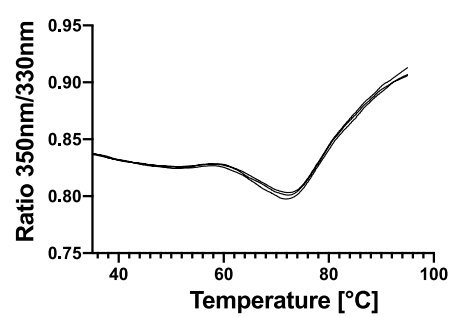

g

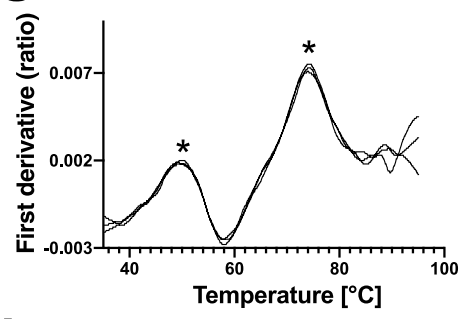

h

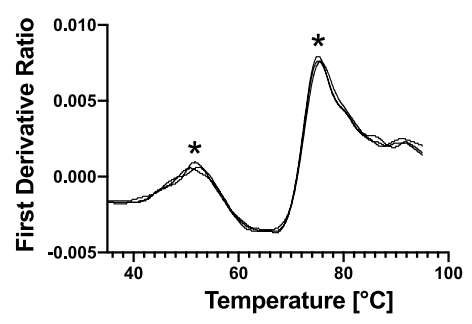

i

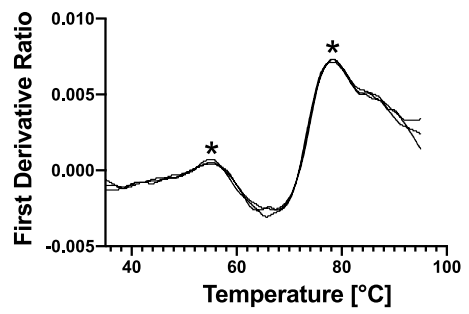

j

\section{Ti value $\left({ }^{\circ} \mathrm{C}\right) \quad$ Ti value $\left({ }^{\circ} \mathrm{C}\right)$}

\begin{tabular}{lcc}
\hline Unmutated & 50.1 & 74.3 \\
u1S2q & 51.7 & 75.2 \\
rS2d & 55.2 & 78.3 \\
\hline Ti, inflection temperature determined in the nCoV buffer \\
(2 mM Tris, pH 8.0,
\end{tabular}

Extended Data Fig. $\mathbf{5}$ | Thermostability of the S protein constructs. a-c, SEC profile of the S-proteins. The dotted lines indicate the portion of the peak that was collected for further studies. The unmutated and u1S2q spikes were run on a Superose 6 Increase $10 / 300$ column, and the rS2d spike was run on an analytical Superose 6 Increase 5/150 column. d-i, Unfolding profile curves obtained by intrinsic fluorescence measurements using Tycho NT. 6. d-f, show ratio between fluorescence at $350 \mathrm{~nm}$ and $330 \mathrm{~nm}$. g-i, plot the first derivative of this ratio. j, Inflection temperatures for the S-proteins. Asterisk mark the inflection temperatures in $(\mathbf{g})$. 


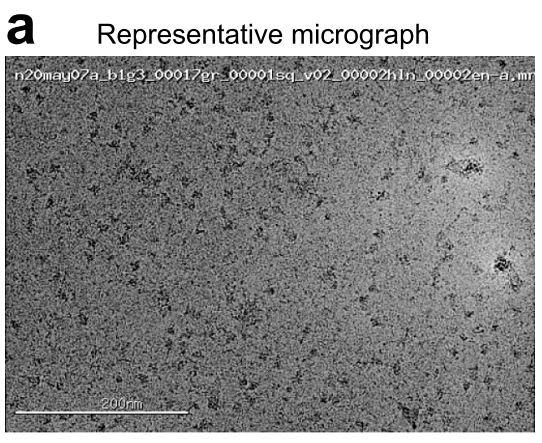

6021 micrographs; $1,313,881$ particles

d

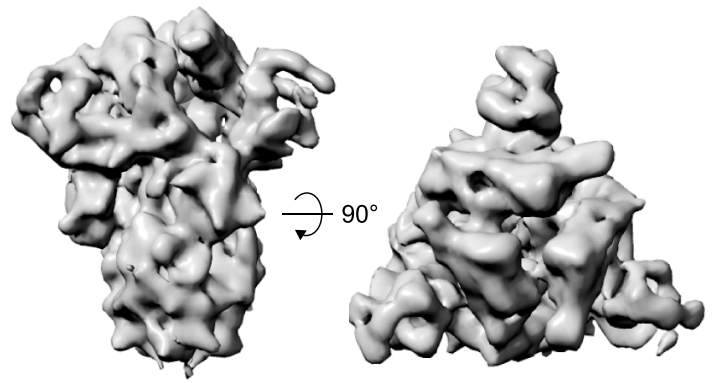

"down" state

C1 symmetry

\section{f}

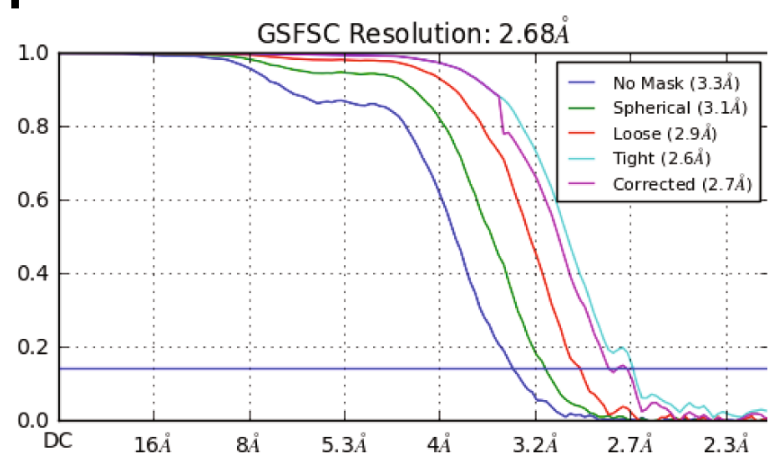

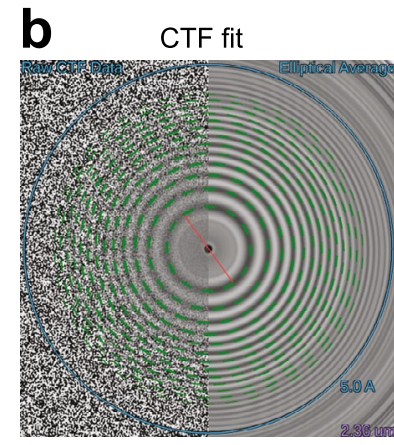

Q
C Representative 2D class averages
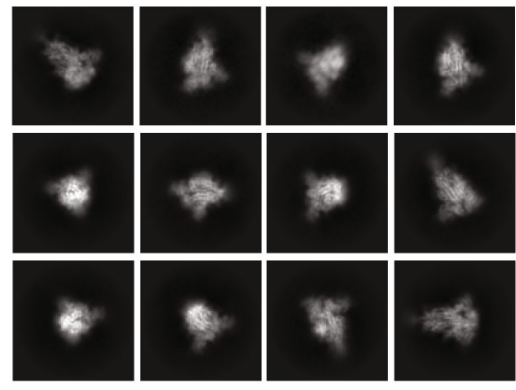

796,589 particles

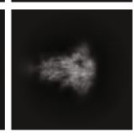

3e

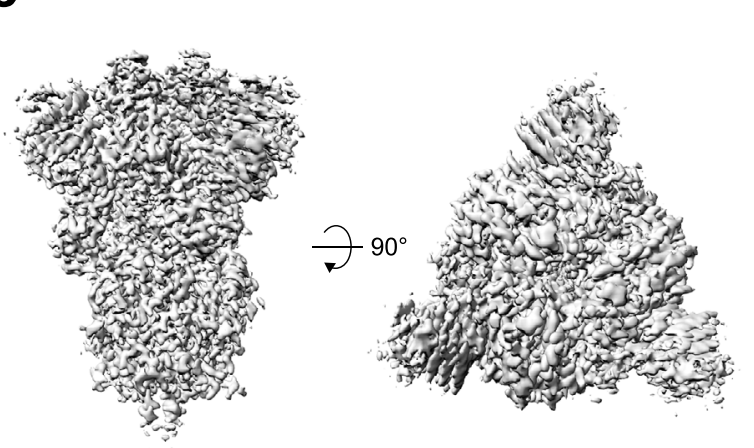

"down" state

367259 particles

C3 symmetry

g

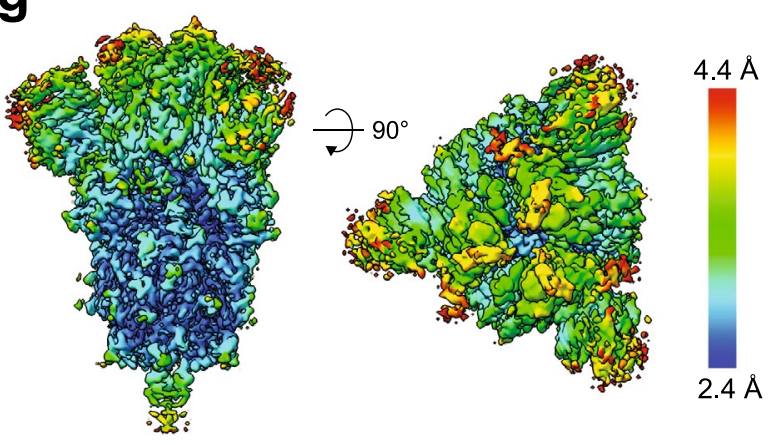

h

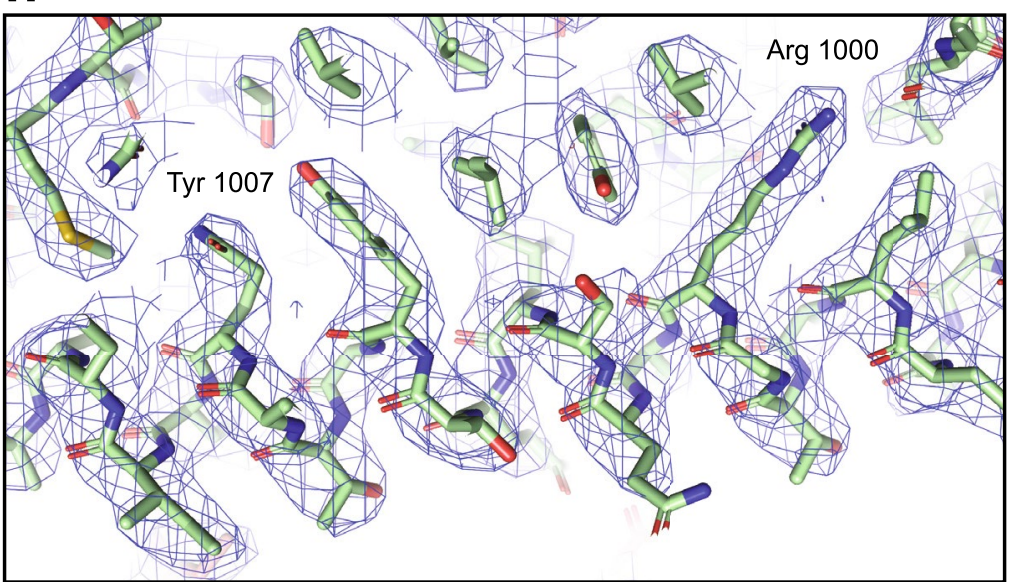

Extended Data Fig. 6 | Cryo-EM data processing details for rS2d. a, Representative micrograph. b, CTF fit c, Representative 2D class averages. d, Ab initio reconstruction. e, Refined map f, Fourier shell correlation curves $\mathbf{g}$, Refined map colored by local resolution. $\mathbf{h}$, Zoomed-in view of the S2 region showing cryo-EM reconstruction as a transparent grey surface, the underlying fitted model in cartoon representation, and residues in ball-and-stick representation. 
a

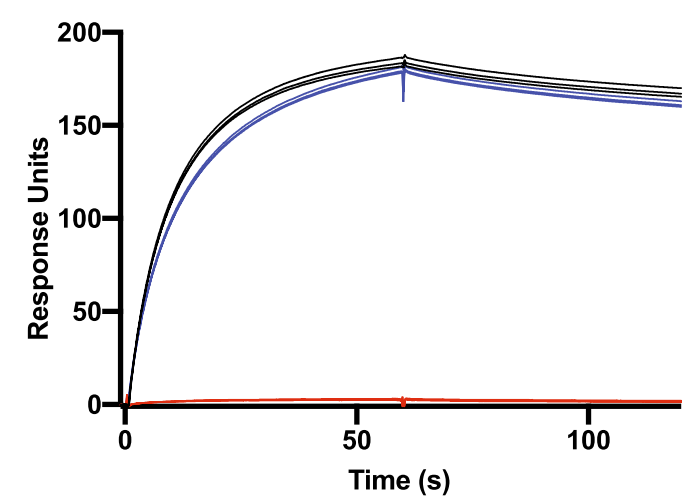

C

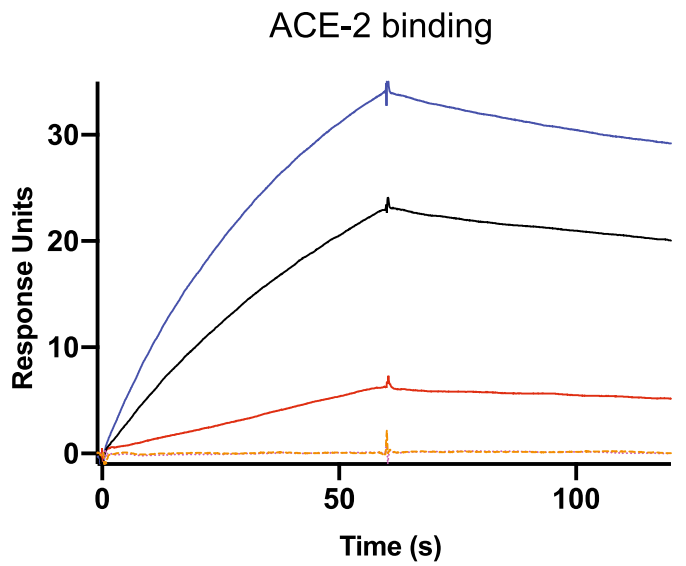

e

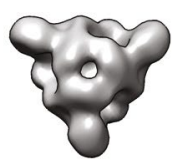

b ACE-2 binding

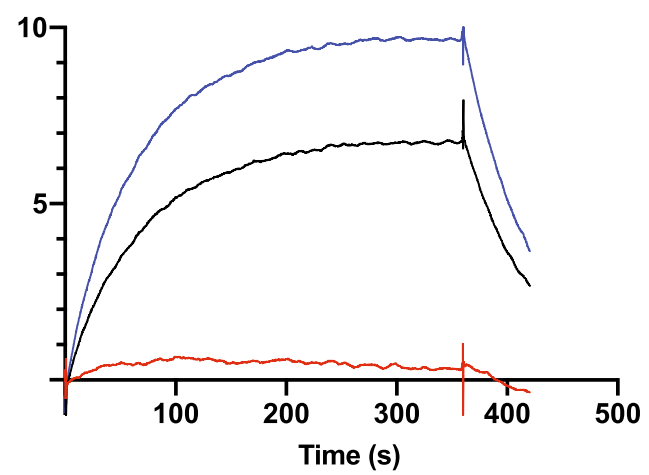

d
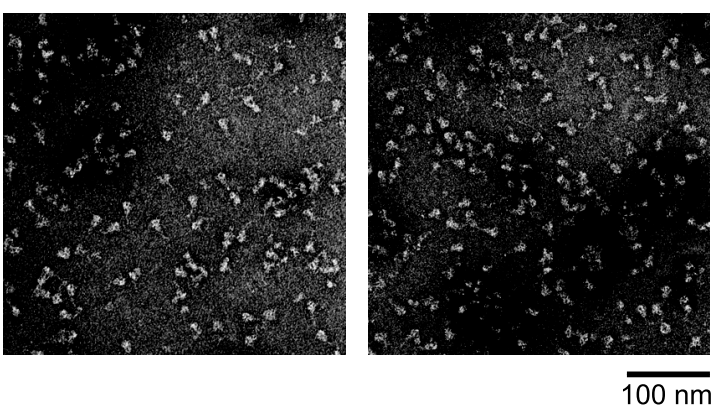

f
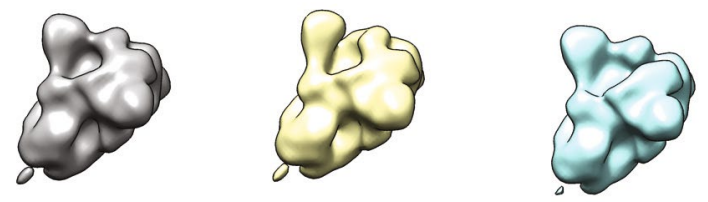
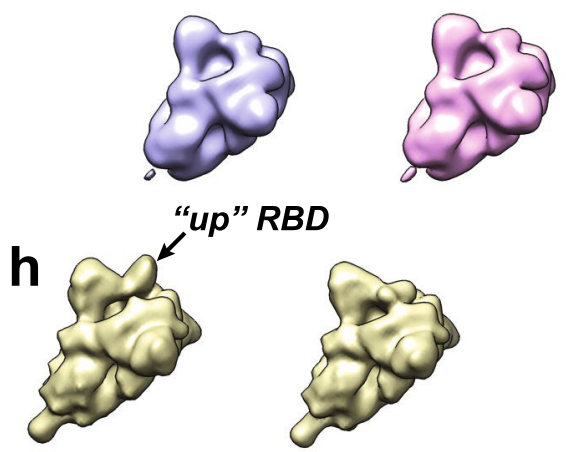

$50 \AA$

Extended Data Fig. 7 | Binding of spike constructs to ACE-2 and RBD-binding antibody CR3022. a, SPR sensorgrams of CR3022 antibody binding to unmutated (black line), u1S2q (blue line) or rS2d (red line) spike captured on a strepdavidin chip. b, SPR sensorgrams of ACE-2 (with C-terminal human Fc tag) to unmutated (black line), u1S2q (blue line) or rS2d (red line) spike captured on a strepdavidin chip. c, SPR sensorgrams of binding of unmutated (black line), u1S2q (blue line) or rS2d (red line) spike to ACE-2 (with C-terminal human Fc tag) captured on an anti-mouse Fc surface. The orange and magenta dotted lines are binding curves for rS2d following negative selection over a CR3022 or ACE-2 column, respectively. d, Representative NSEM micrographs of the flow-through after negative selection of the rS2d sample through an ACE-2 column (left) and CR3022 column (right). e, Top views and $\mathbf{f}$, side views of 3D classes of particles from the dataset from the CR3022 column-eluted sample. C1 symmetry was used during classification. For comparison, images from cryo-EM maps low-pass filtered to $20 \AA$ of the 1-RBD 'up' state (EMD-21457) and 3-RBD 'down' state (EMD-21452) are shown in figures $\mathbf{g}$, top view and $\mathbf{h}$, side view. 
a

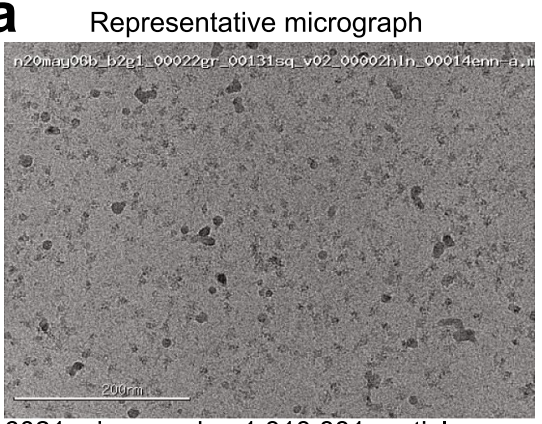

6021 micrographs; $1,313,881$ particles

d

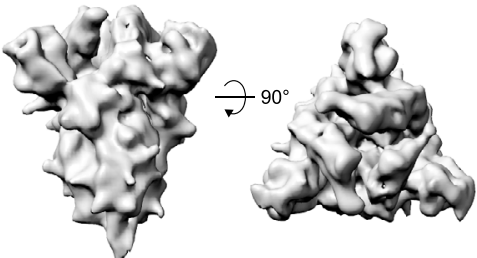

"down" state

192430 particles

C1 symmetry

g

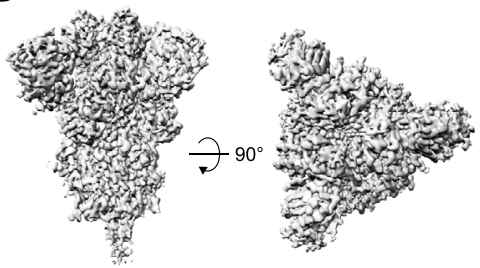

"down" state

192430 particles

$3.2 \AA$, C3 symmetry

j

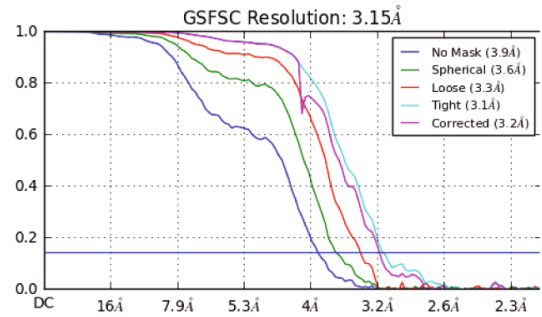

b

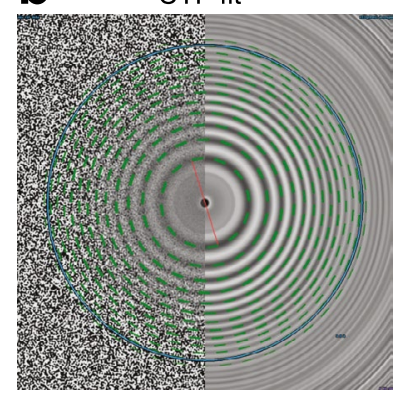

e

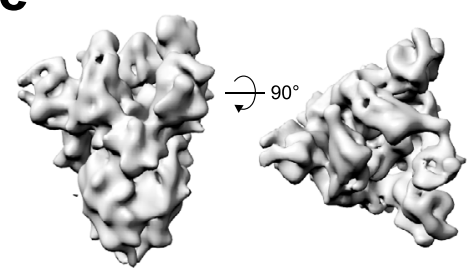

"1-up" state

255013 particles

C1 symmetry

h

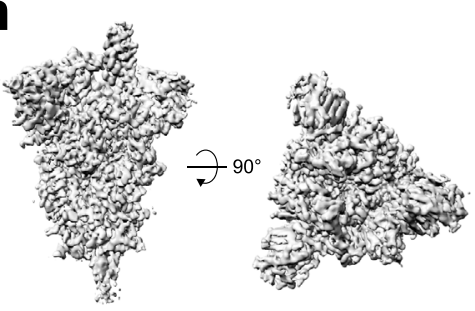

"1-up" state

255013 particles

$3.3 \AA$, C1 symmetry

\section{k}

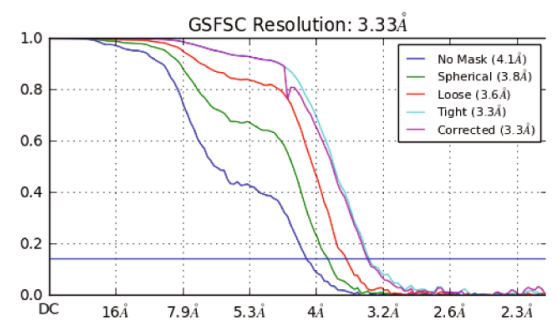

C Representative 2D class averages

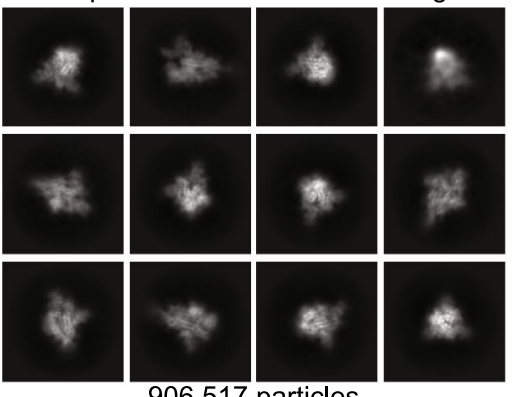

f

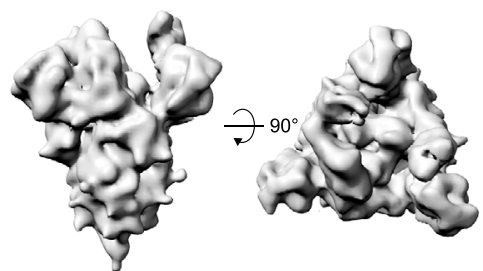

"2-up" state

133957 particles

C1 symmetry

i

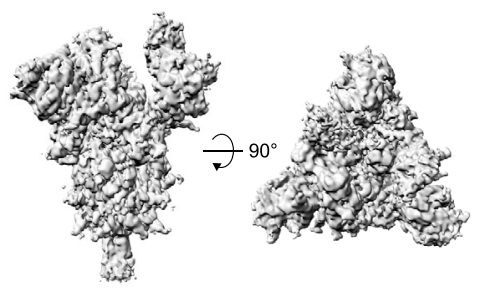

"2-up" state

133957 particles

3.6 Å, C1 symmetry

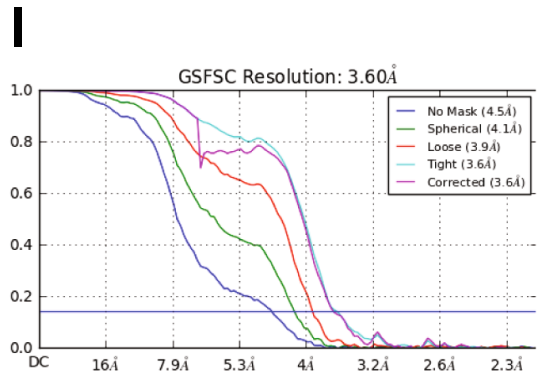

Extended Data Fig. 8 | Cryo-EM data processing details for u1s2q. a, Representative micrograph. b, CTF fit c, Representative 2D class averages. d-f, $A b$ initio reconstructions for the (d) 'down' state, (e) '1-up' state and (f) '2-up' state. g-i, Refined maps for the (g) 'down' state, (h) '1-up' state and (i) '2-up' state. $\mathbf{j}-\mathbf{I}$, Fourier shell correlation curves for the (j) 'down' state, (e) '1-up' state and (f) '2-up' state. 
a

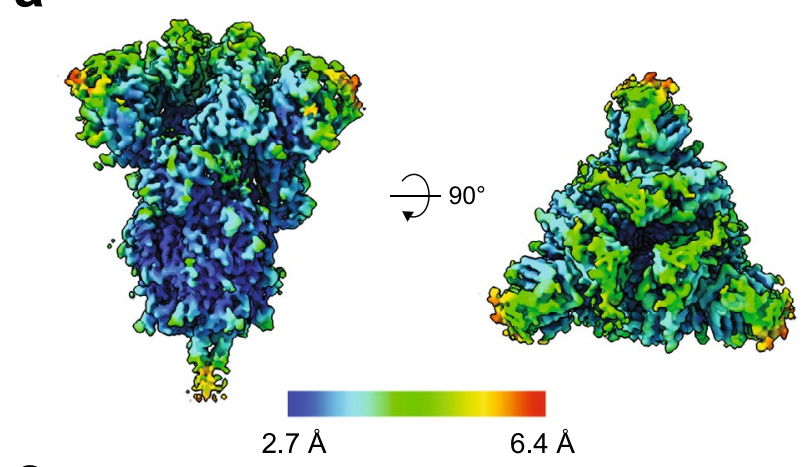

C

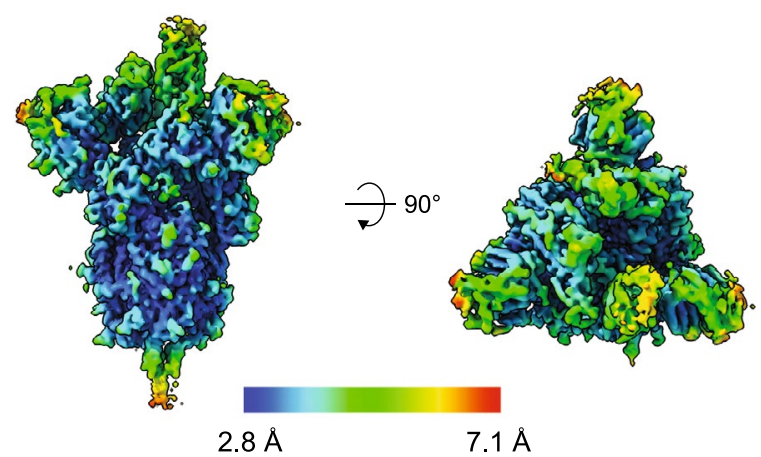

e

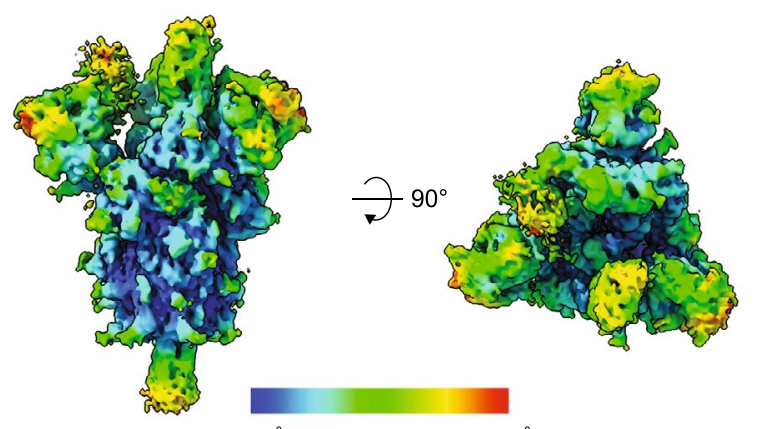

b

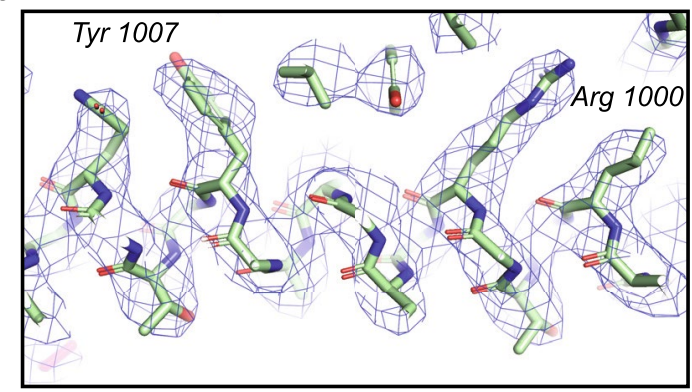

d

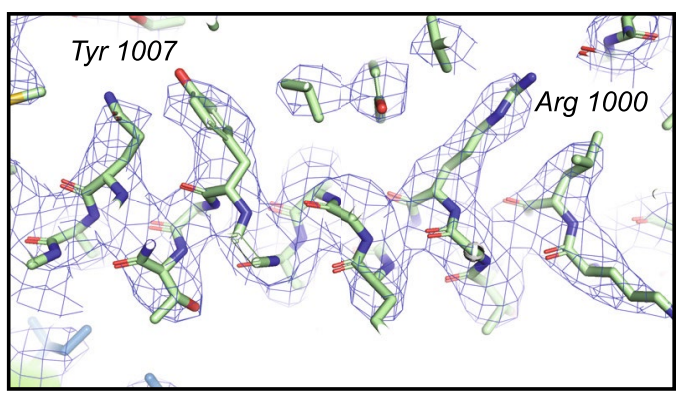

f

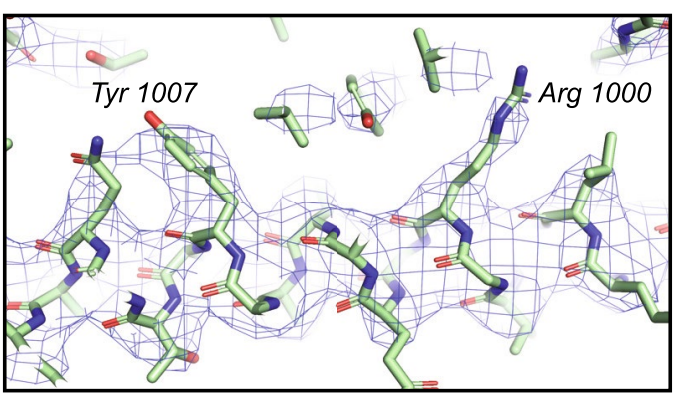

Extended Data Fig. 9 | Local map resolution for u1s2q. a, Refined cryo-EM maps colored by local resolution and $\mathbf{b}$, Zoom-in image showing region in the S2 domain with the cryo-EM map shown as a transparent surface and underlying fitted model in cartoon representation, with residues shown as balls and sticks. c, and d, Same information as presented in panels (a) and (b) but for the 1-RBD 'up' state. (e) and (f) Same information as presented in panels (a) and (b) but for the 2-RBD 'up' state. 
a

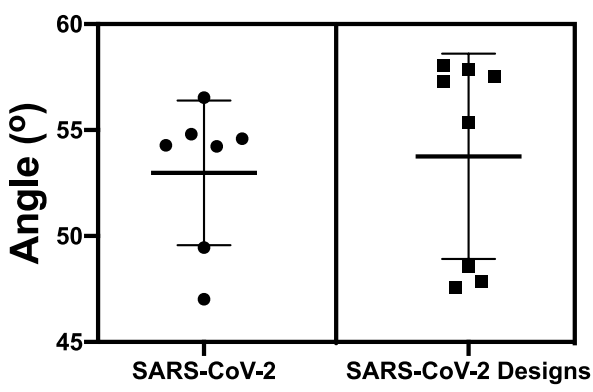

b
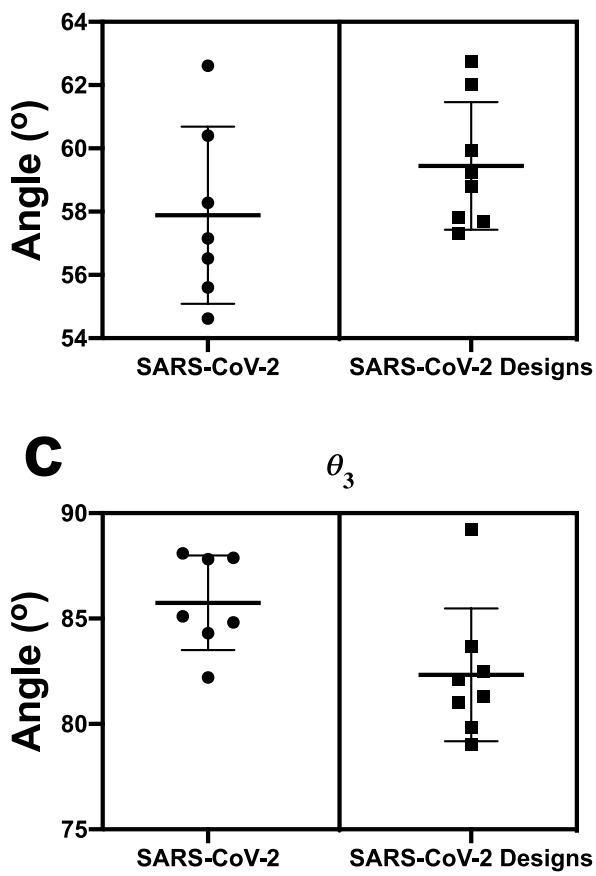

d

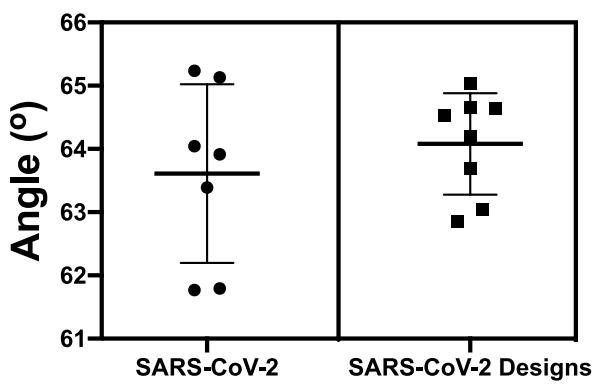

$\theta_{5}$

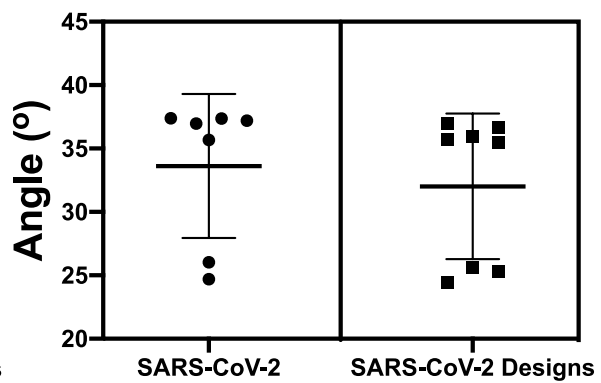

f

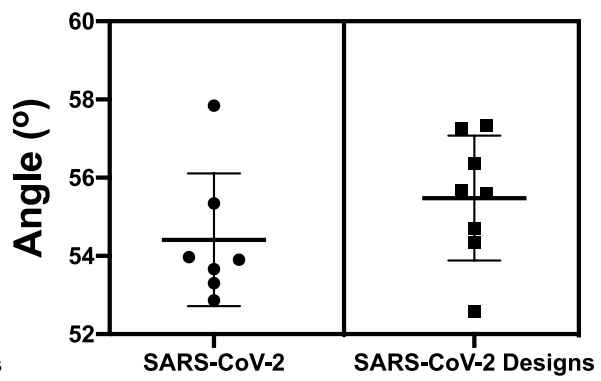

g

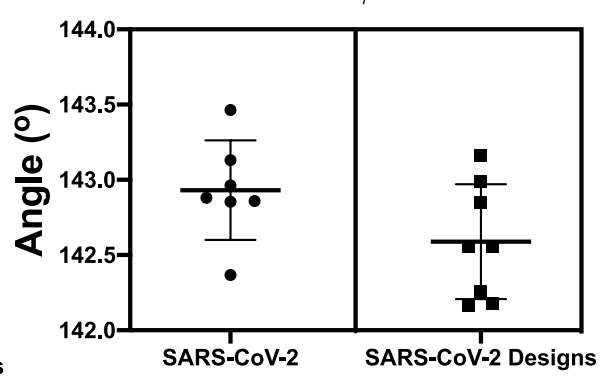

h

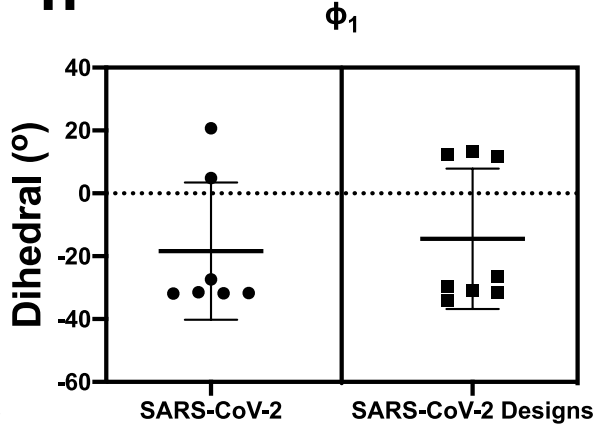

h $\phi_{2}$
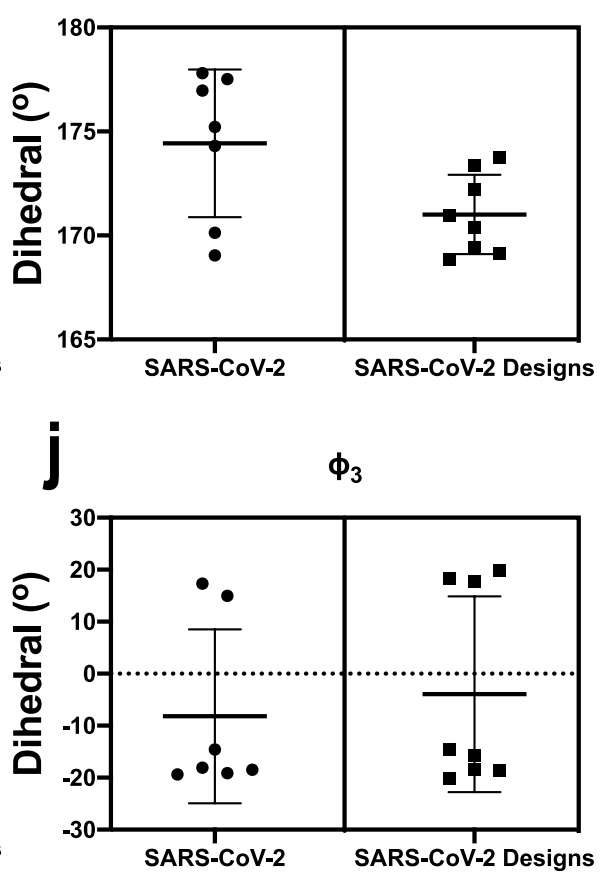

K $\phi_{4}$

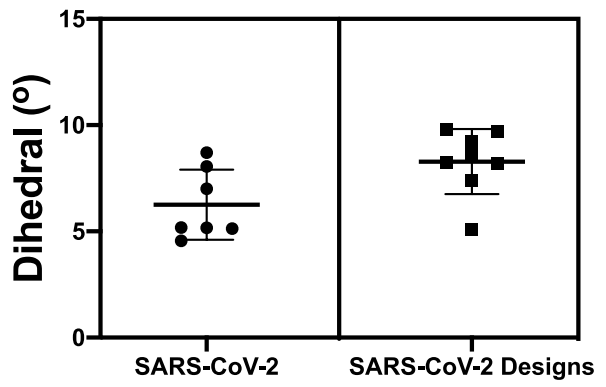

Extended Data Fig. 10 | $\beta$-CoV Vector Analysis of SARS-2 and SARS-2 Designs. a-k, Angles and dihedrals for SARS-2 structures and SARS-2 designs depicted in Fig. 1c. Individual data points are shown as circles or squares; lines denote mean and s.d. 


\section{Reporting Summary}

Nature Research wishes to improve the reproducibility of the work that we publish. This form provides structure for consistency and transparency in reporting. For further information on Nature Research policies, see our Editorial Policies and the Editorial Policy Checklist.

\section{Statistics}

For all statistical analyses, confirm that the following items are present in the figure legend, table legend, main text, or Methods section.

n/a $\mid$ Confirmed

\The exact sample size $(n)$ for each experimental group/condition, given as a discrete number and unit of measurement

$\square$ A statement on whether measurements were taken from distinct samples or whether the same sample was measured repeatedly

$\triangle \square$ The statistical test(s) used AND whether they are one- or two-sided

Xnly common tests should be described solely by name; describe more complex techniques in the Methods section.

Х A description of all covariates tested

Х $\square$ A description of any assumptions or corrections, such as tests of normality and adjustment for multiple comparisons

A full description of the statistical parameters including central tendency (e.g. means) or other basic estimates (e.g. regression coefficient) AND variation (e.g. standard deviation) or associated estimates of uncertainty (e.g. confidence intervals)

Х For null hypothesis testing, the test statistic (e.g. $F, t, r$ ) with confidence intervals, effect sizes, degrees of freedom and $P$ value noted Give $P$ values as exact values whenever suitable.

Х $\square$ For Bayesian analysis, information on the choice of priors and Markov chain Monte Carlo settings

Х $\square$ For hierarchical and complex designs, identification of the appropriate level for tests and full reporting of outcomes

Х $\square$ Estimates of effect sizes (e.g. Cohen's d, Pearson's $r$ ), indicating how they were calculated

Our web collection on statistics for biologists contains articles on many of the points above.

\section{Software and code}

Policy information about availability of computer code

Data collection Provide a description of all commercial, open source and custom code used to collect the data in this study, specifying the version used OR state that no software was used.

Data analysis Pymol, VMD, cryoSPARC, UCSF Chimera, Coot, Schrodinger, Phenix, R, Biacore T200 Evaluation Software

For manuscripts utilizing custom algorithms or software that are central to the research but not yet described in published literature, software must be made available to editors and

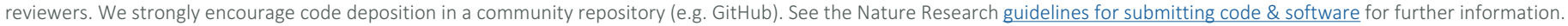

\section{Data}

Policy information about availability of data

All manuscripts must include a data availability statement. This statement should provide the following information, where applicable:

- Accession codes, unique identifiers, or web links for publicly available datasets

- A list of figures that have associated raw data

- A description of any restrictions on data availability 
Please select the one below that is the best fit for your research. If you are not sure, read the appropriate sections before making your selection.
$\bigotimes$ Life sciences
Behavioural \& social sciences
Ecological, evolutionary \& environmental sciences

For a reference copy of the document with all sections, see nature.com/documents/nr-reporting-summary-flat.pdf

\section{Life sciences study design}

All studies must disclose on these points even when the disclosure is negative.

Sample size n/a
Data exclusions n/a
Replication n/a
Randomization $n$ n/a
Blinding

\section{Reporting for specific materials, systems and methods}

We require information from authors about some types of materials, experimental systems and methods used in many studies. Here, indicate whether each material, system or method listed is relevant to your study. If you are not sure if a list item applies to your research, read the appropriate section before selecting a response.

\begin{tabular}{l|l} 
Materials \& experimental syste \\
\hline n/a & Involved in the study \\
\hline & $\bigotimes$ Antibodies \\
$\square$ & $\bigotimes$ Eukaryotic cell lines \\
$\square$ & $\square$ Palaeontology and archaeology \\
$\square$ & $\square$ Animals and other organisms \\
$\searrow$ & $\square$ Clinical data \\
$\square$ & $\square$ Dual use research of concern
\end{tabular}

\begin{tabular}{l|l} 
Methods \\
\hline n/a & Involved in the study \\
$\searrow$ & $\square$ ChIP-seq \\
$\square$ & $\square$ Flow cytometry \\
$\square$ & $\square$ MRI-based neuroimaging
\end{tabular}

Antibodies

$\begin{array}{ll}\text { Antibodies used } & \text { CR3022 IgG } \\ \text { Validation } & \begin{array}{l}\text { Cloned in-house and verified by DNA sequencing. Purified antibody validated using SDS-PAGE and binding to SARS-CoV-2 spike } \\ \text { ectodomain and SARS-CoV-2 RBD by flow cytometry and ELISA. }\end{array}\end{array}$

\section{Eukaryotic cell lines}

Policy information about cell lines

Cell line source(s)

Authentication

Mycoplasma contamination

Commonly misidentified lines (See $\underline{I C L A C}$ register)

Freestyle 293 cells from Invitrogen was used to express S-protein trimers

Manufacturer of the cell line provides authentication information on their website

Mycoplasma contamination screening is described on the manufacturer's website

$\mathrm{n} / \mathrm{a}$ 